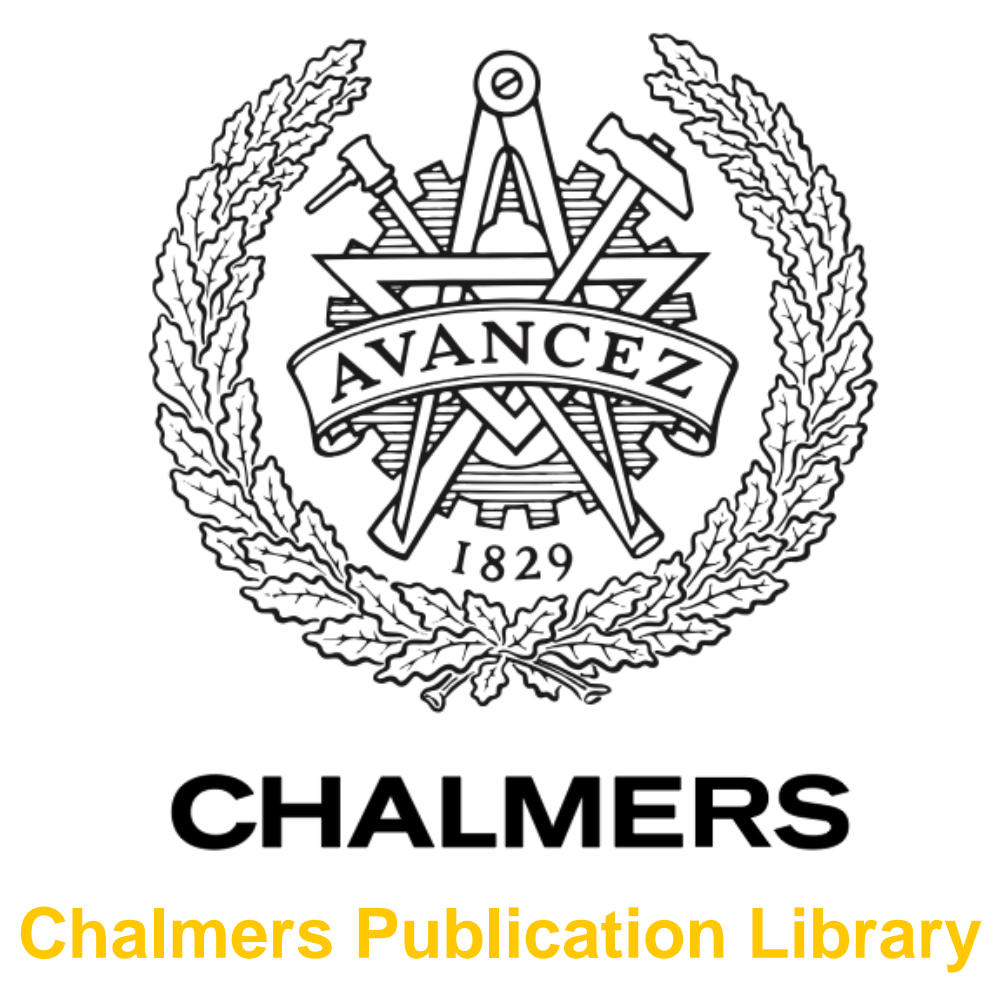

\author{
Optimized brake-based control of path lateral deviation for mitigation of secondary \\ collisions
}

This document has been downloaded from Chalmers Publication Library (CPL). It is the author's version of a work that was accepted for publication in:

Proceedings of the Institution of Mechanical Engineers Part D - Journal of Automobile Engineering (ISSN: 0954-4070)

Citation for the published paper:

Yang, D. ; Gordon, T. ; Jacobson, B. (2011) "Optimized brake-based control of path lateral deviation for mitigation of secondary collisions". Proceedings of the Institution of Mechanical Engineers Part D - Journal of Automobile Engineering, vol. 225(D12), pp. 1587-1604.

http://dx.doi.org/10.1177/0954407011412856

Downloaded from: http://publications.lib.chalmers.se/publication/156560

Notice: Changes introduced as a result of publishing processes such as copy-editing and formatting may not be reflected in this document. For a definitive version of this work, please refer to the published source. Please note that access to the published version might require a subscription.

Chalmers Publication Library (CPL) offers the possibility of retrieving research publications produced at Chalmers University of Technology. It covers all types of publications: articles, dissertations, licentiate theses, masters theses, conference papers, reports etc. Since 2006 it is the official tool for Chalmers official publication statistics. To ensure that Chalmers research results are disseminated as widely as possible, an Open Access Policy has been adopted.

The CPL service is administrated and maintained by Chalmers Library. 


\title{
Optimized brake-based control of path lateral deviation for mitigation of secondary collisions
}

\author{
D Yang $^{1^{*}}$, T J Gordon ${ }^{2}$, B Jacobson ${ }^{3}$, M Jonasson ${ }^{3}$, and M Lidberg ${ }^{1}$ \\ ${ }^{1}$ Department of Applied Mechanics, Chalmers University of Technology, Gothenburg, Sweden \\ ${ }^{2}$ Department of Mechanical Engineering, University of Michigan, Ann Arbor, MI, USA \\ ${ }^{3}$ Department of Active Safety and Chassis, Volvo Car Corporation, Gothenburg, Sweden
}

The manuscript was received on 15 October 2010 and was accepted after revision for publication on 17 May 2011.

DOI: $10.1177 / 0954407011412856$

\begin{abstract}
This paper considers brake-based lateral control of a passenger vehicle, for reducing secondary collision risk following an initial impact in a traffic accident. Since secondary collisions are associated with deviations from the original travel path, the control problem is formulated via brake control sequences that minimize lateral path deviation. Optimal sequences are found not to conform to any simple control mode; sometimes all brakes are released, sometimes all wheels are locked, or the brakes may be applied in differential mode. In general, the optimal strategy combines several such actuation modes, and analysis shows it is related to the utilization of instantaneous vehicle force and moment capacity, indicating that a closed-loop control strategy may be developed based on the real-time estimation of tyre force limits during the post-impact event. Yaw motion control is related to response discontinuity and multiple equilibria found in the optimal response - a small change in initial yaw velocity generates large changes in the ensuing vehicle motion and thus in the aimed equilibrium point of the vehicle's orientation. Overall it is found that braking control strongly influences the post-impact path of the impacted vehicle, and may therefore form the basis of a practical system for avoiding secondary collisions in future traffic accidents.
\end{abstract}

Keywords: active safety, collision avoidance, path control, braking, post-impact, optimization, vehicle dynamics

\section{INTRODUCTION}

Vehicle traffic safety attracts considerable attention from all perspectives, given the continuing high numbers of accidents registered in road traffic statistics. One type of accident is gradually increasing according to recent accident statistics studies Multiple-Event Accidents (MEAs) [1]. These are characterized by having at least one vehicle subjected to more than one harmful event, such as collision with another vehicle. Statistics show that

\footnotetext{
*Corresponding author: Department of Applied Mechanics, Vehicle Dynamics, Chalmers University of Technology, SE-412 96, Gothenburg, Sweden. email: derong.yang@chalmers.se
}

MEAs comprise up to a third of all passenger vehicle accidents [2-4], and human injury levels in MEAs are higher than in Single-Collision Event [3-5]. Studies of more recent accident statistics have shown that MEAs have a threefold increase in risk for severe injury and fourfold increase for fatal injury, as compared to Single Event Accidents (SEAs) [6]. Most MEAs experience the secondary event due to excessive lateral deviation from the road or lane centre after an initial collision [2]. The secondary event may be a collision with a road-side stationary object or another moving vehicle, or it may be a rollover event. A previous study on the estimation of potential safety benefits gained from post-impact (PI) interventions found that the majority of MEAs occurred on straight continuous roads 
or at intersections [7]; it was concluded that if lateral deviations were to be reduced, in many cases it is possible to mitigate or completely avoid secondary events. Therefore, the ability to minimize the PI path lateral deviation $Y(t)$ can be greatly beneficial to road traffic safety, provided significant controlled changes in path are feasible.

Vehicle dynamic control using brakes is well known in terms of classical electronic stability control (ESC) systems, which infer and follow the driver's intended yaw rate within the limits of various stability criteria [8-12]. Some previous studies on controlling PI vehicle dynamics $[\mathbf{5}, \mathbf{1 3}]$ follow a similar approach, seeking to instantaneously minimize any large PI yaw rate; in one study the system applies automatic longitudinal deceleration on all four wheels via the brakes [14]. In reference [5] a stabilization controller was developed to attenuate excessive vehicle yaw rate via differential braking after a set of angled rear-end impacts; its path control was achieved by first recovering stability then allowing the driver to steer back to the road centre. In reference [13] a yaw moment optimization algorithm was developed to reduce yaw rate in various skidding and spinning motions, and the tuning of the individual wheel brake slip and front axle steering angles was presented. In reference [14], a function named Secondary Collision Mitigation (SCM) retrieves information of the first impact from the airbag system and sends this to ESC control units which command braking actuation. Another approach, from the California PATH programme [15], also considered that a minor or moderate collision will not disable the actuators of a vehicle, and that a strategy of controlling the vehicle trajectories would mitigate the accident consequences significantly. In that work, steering controllers were developed using a linear model of the vehicle lateral dynamics; however, the PI dynamics were limited to mild cases, considering only rear-end collisions with small offsets.

In this paper the authors formulate the problem of secondary event avoidance as a path optimization problem: to minimize lateral path deviation from the pre-impact trajectory using active control of brakes. This new approach is expected to provide a very different type of control compared to those mentioned above, especially because there is no explicit requirement to minimize yaw rate; indeed, the possibility exists to exploit the yaw degree of freedom for maximum benefit to reduce path deviation. Also distinct from ESC interventions, the new path control strategy does not derive its reference from the driver, but from the global geometry. In this paper, a straight road is assumed and that the reference path is parallel to the driver's original path before impact. This is in keeping with a new generation of on-market active safety systems relying on environment sensors (e.g. Collision Mitigation by Braking, City Safety [16]), including popularly investigated lane-keeping [17] and collision avoidance systems using active brake and/or steer interventions [18-23].

In order to reduce lateral deviation, it is clear that a resultant force is required perpendicular to the original intended path. This force is to be controlled by brake actuation at each wheel, and derives from both longitudinal and lateral forces at the tyres. Tyre forces available at any instant depend on normal loads and tyre-road friction, as well as slip angles at the individual wheels, and in turn these depend on steer angles and vehicle kinematics, especially yaw rate and body side slip angle. Part of the control problem is therefore in cascading a desired resultant vehicle force down to the level of the individual brake actuators. Such problems of brake torque apportionment has been extensively studied in the area of Direct Yaw Control (DYC), where different tyre longitudinal forces between the left and right are properly generated to control vehicle motion via a yaw moment reference signal $[\mathbf{2 4}$, 25]. Apportionment concepts are to be used here for vehicle path control, relating available resultant global forces to vehicle kinematics in a variety of PI scenarios.

The paper is structured as follows. In section 2 the vehicle models are defined and an optimization scheme is formulated. Sample results are presented and analysed in section 3, while in section 4 the strategies used by the optimized brake controller are explored via relationships between applied braking torques and the force-moment availability at the vehicle level. In section 5 the full range of brake actuation modes is determined using phase-plane analysis, and conclusions are given in section 6 .

\section{METHOD}

The approach in this paper is to apply general optimization techniques to the PI path control problem, with emphasis on finding out the feasible effects of brake application and the mechanisms that are most effective. Hereby, the underlying hypothesis is that minimizing a cost function, defined to penalize the vehicle's maximum lateral deviation $Y_{\max }$ from the original intended lane, may be effective in controlling path deviations. The focus is to directly optimize braking sequences rather than developing control algorithms and optimizing parameters; this 
is so performance limits can be determined and a deeper understanding of the dynamic principles can be established. The resulting performance will then be compared with simpler control modes such as full braking to wheel-lock.

\subsection{Vehicle simulation model}

The vehicle model used in the simulation is a 3DOF planar two-track model with three velocity states relative to the ground $x-y$ coordinate. In this study it is assumed that the driver, or some other actuators, constrain the road wheels to zero steer angle during the events. Using the notation as given in Appendix 1, the equations of motion are

$$
\begin{aligned}
& m\left(\dot{v}_{x}-v_{y} \dot{\psi}\right)=F_{x \mathrm{fl}}+F_{x \mathrm{fr}}+F_{x \mathrm{rl}}+F_{x \mathrm{rr}} \\
& m\left(\dot{v}_{y}+v_{x} \dot{\psi}\right)=F_{y \mathrm{fl}}+F_{y \mathrm{fr}}+F_{y \mathrm{rl}}+F_{y \mathrm{rr}} \\
& I_{z z} \ddot{\psi}=l_{\mathrm{f}}\left(F_{y \mathrm{fl}}+F_{y \mathrm{fr}}\right)-l_{\mathrm{r}}\left(F_{y \mathrm{rl}}+F_{y \mathrm{rr}}\right) \\
& \quad+\frac{t}{2}\left(F_{x \mathrm{fr}}-F_{x \mathrm{fl}}\right)+\frac{t}{2}\left(F_{x \mathrm{rr}}-F_{x \mathrm{rl}}\right)
\end{aligned}
$$

This simple 3-DOF model is selected since, during optimization, it converges quickly compared to a corresponding 7-DOF vehicle model which additionally includes wheel rotational dynamics. The 7-DOF model was however used to validate results of a number of optimizations. It is found that the optimal braking sequences, resulting vehicle responses, as well as optimization performance (cost versus iteration) are all very similar between the two models. A typical case is illustrated in Fig. 1, using the same starting conditions in optimization and comparing 3DOF and 7-DOF model behaviours.

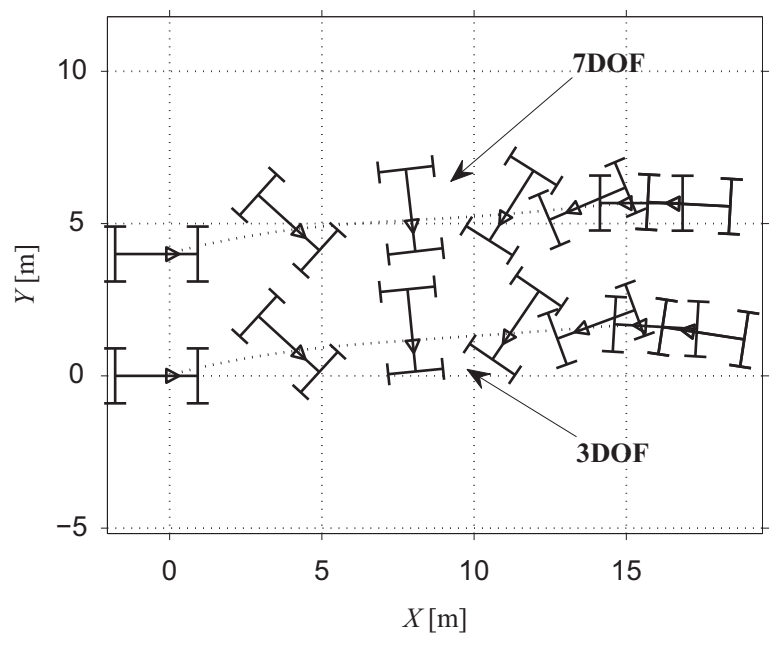

(a) Optimized Path Comparison: $v_{\mathrm{PI}}=15 \mathrm{~m} / \mathrm{s}$, $\beta_{\mathrm{PI}}=15 \mathrm{deg}, \dot{\psi}_{\mathrm{PI}}=-2.5 \mathrm{rad} / \mathrm{s}$.
A simplified version of Magic Formula (MF) tyre model with $F_{x i}$ as input variable [26], is implemented

$$
\left\{\begin{array}{c}
F_{y i}\left(\alpha_{i}\right)=-D_{i} \cdot \sin \left\{C _ { i } \cdot \operatorname { a r c t a n } \left[B_{i} \cdot \alpha_{i}-E_{i} .\right.\right. \\
\left.\left.\quad\left(B_{i} \cdot \alpha_{i}-\arctan \left(B_{i} \cdot \alpha_{i}\right)\right)\right]\right\} \\
D_{i}=\sqrt{\left(\mu_{i} \cdot F_{z i}\right)^{2}-F_{x i}^{2}}
\end{array}\right.
$$

$D_{i}$ is the peak lateral tyre force formulated as a function of $F_{x i}$, as well as friction coefficient and vertical load. The tyre model parameters are tuned in accordance with representative data from a passenger car tyre [7]. In extreme spinning and sliding motions after an impact, the tyre side slip angle $\alpha$ commonly exceeds $90^{\circ}$, and the tyre model is thus extended (see Fig. 2).

The vehicle model is further validated by comparing vehicle dynamics states and tyre forces with those from simulations in veDYNA [7].

\subsection{Objective function}

The objective function for optimization is chosen to be relevant to typical severity probability of secondary events in an MEA, occurring on a straight road. It is based on both the lateral deviation from the intended path and the time duration of this deviation [27]. The objective function is defined as

$$
C=\sqrt[4]{\frac{\int_{0}^{t_{\text {end }}} Y^{4} \cdot \mathrm{d} t}{t_{\text {end }}}}
$$

Here, a $p$-norm formulation is used, with $p=4$ chosen so the cost is biased towards $Y_{\max }=\max (|Y|)$.

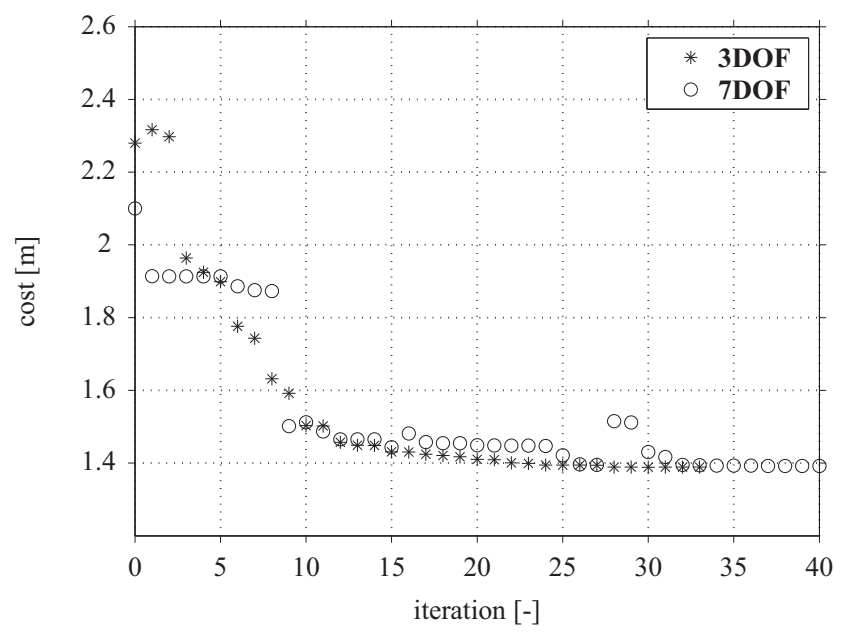

(b) Cost versus iteration: $v_{\mathrm{PI}}=15 \mathrm{~m} / \mathrm{s}$, $\beta_{\mathrm{PI}}=15 \mathrm{deg}, \dot{\psi}_{\mathrm{PI}}=-2.5 \mathrm{rad} / \mathrm{s}$.

Fig. 1 Comparison of 3-DOF and 7-DOF model in optimization 


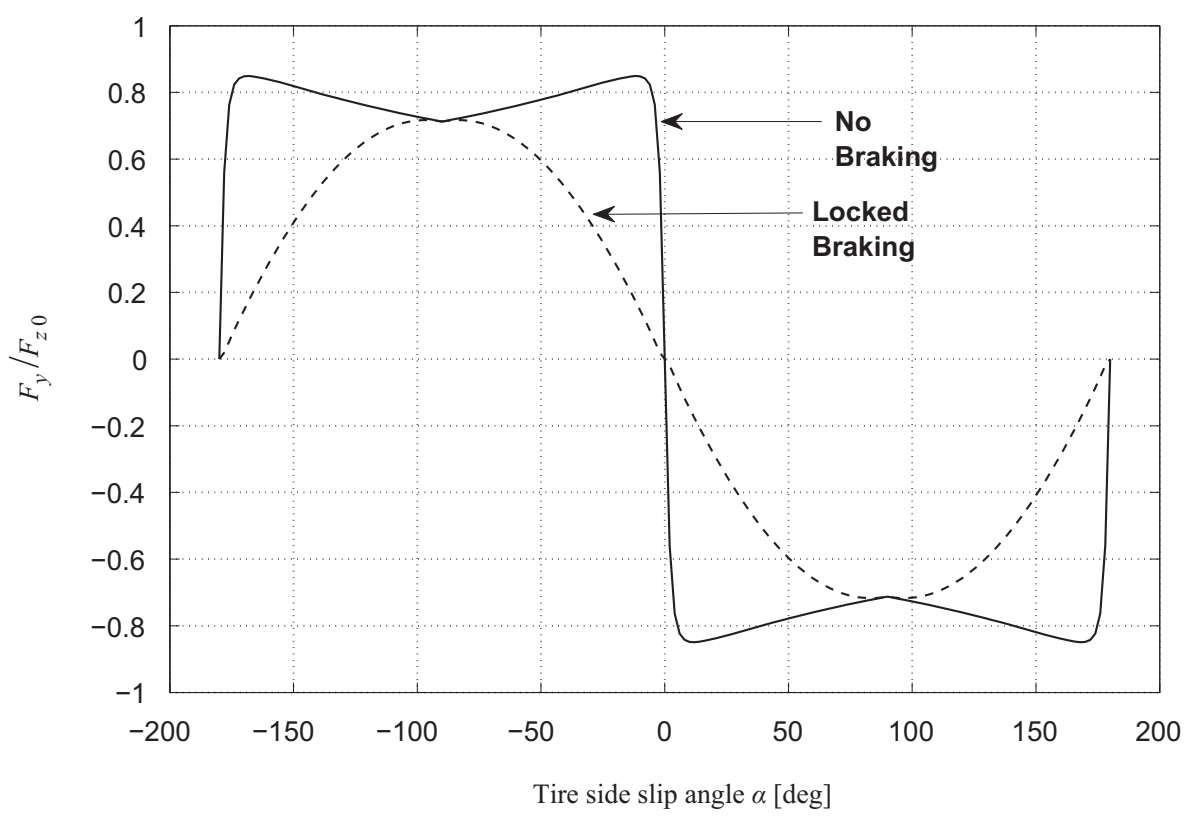

Fig. 2 Simplified MF tyre model, normalized lateral tyre force versus tyre side slip angle. (Locked braking: $F_{x i}=\mu_{i} F_{z i} \cdot \cos \left(\alpha_{i}\right)$.)

This is primarily used because $Y^{4}$ is a smooth function expected to be numerically well-behaved, avoiding troublesome discontinuities while still approximating the optimal controls for minimizing $Y_{\max }$. It is also justified from a practical standpoint - the likelihood and severity of an impact is expected to increase with the overall path lateral deviation, and not exclusively dependent on the location of the car at one single time instant. The event simulation time $t_{\text {end }}$ is set to $1.8 \mathrm{~s}$, starting from the end of initial impact. This is consistent with accident analysis, which shows the time to the second collision is usually within $2 \mathrm{~s}$ [7], and avoids the complication that the vehicle may completely come to rest before the end of the simulation. It is noted that optimization results from various PI initial conditions confirm that the cost function follows $Y_{\max }$ quite well: the higher the cost, the higher $Y_{\max }$ is found to be.

\subsection{Optimization}

Numerical optimization is performed using a standard gradient-based method, as implemented in the MATLAB function fmincon [28]. This determines a constrained minimum of the objective function, including operating bounds on control variables, in this case the brake pad application forces [27]. It is assumed that individual wheels can be braked dynamically after the initial impact; brake cylinder pressures are individually modulated according to a strategy that is to be determined via the optimization. The brake actuation sequence on each wheel is set to start from zero and vary every $0.18 \mathrm{~s}$ in 10 equal time intervals, with linear interpolation between the chosen levels. An upper bound on applied brake pad force or torque is required. Here $10 \mathrm{kN}$ is used as a reasonable value for this bound, based on the legitimate requirements on the hydraulic pressure limit of the brake cylinder in a passenger car [29]. These are then transformed to give tyre longitudinal forces in the tyre-ground contact patches, subject to road friction limits. Since there is no guarantee of finding the global optimum, starting conditions for the braking sequence optimization are varied. Three simple control strategies were used for this: (a) several randomized sequence of light braking pulses, (b) differential braking between left and right wheels in the sense that opposes the instantaneous yaw velocity, (c) full braking to wheel lock on all wheels. On average, five randomized sequences were used in (a). So an estimate of the global optimum is captured by selecting the result with lowest cost; in all cases it was confirmed that the cost from at least one other starting condition closely matched this minimum value.

\section{EFFECTIVENESS OF OPTIMAL BRAKE INTERVENTIONS}

The initial objective is to determine whether worthwhile control can be applied: is it possible to significantly influence the vehicle path under PI conditions using only brake interventions? The 
authors consider three different PI conditions, all of which are within kinematic ranges seen in accident databases [2]. The first case results from a side impact to the front of the mass centre, generating high side slip at the front axle; the second is from a side impact behind the mass centre, with corresponding high side slip at the rear axle. These events involve large PI yaw rates. In contrast, the third event has relatively low PI yaw rate, with the impact point a smaller distance in front of the mass centre. The PI yaw angle is assumed zero here. This corresponds to assuming an instantaneous (rigid body) impact has occurred, while the impacted vehicle was initially travelling parallel to the original travel lane. In practice, the impact involves deformable bodies and the PI kinematic conditions depend on the duration of the impact and the details of momentum and energy transfer between the vehicle and its collision partner. However, this assumption is done without great loss of generality for the optimal control strategies found in the present paper. A brief sensitivity study with realistic non-zero yaw angle at zero time instant verified that it gives qualitatively the same optimal response. The PI initial kinematics are summarized in Table 1, together with an additional case considered later in the paper.

For each of the three cases the effect of the computed optimal control sequence is determined, as well as comparisons with three simple forms of control: (a) no braking, (b) full braking to lock all wheels, and (c) a stabilizing yaw motion controller operating via differential braking (see Appendix 2). These three are similar to those mentioned in section 2 for initialization of the optimization process, though here the randomized braking is replaced with an uncontrolled vehicle, and a yaw angle component is introduced to the yaw motion controller. Controller (a) mimics the deactivation of all active safety systems after collisions and controller (b) is similar to the SCM function mentioned above; controller (c) is chosen to attenuate yaw deviations in a way that is broadly similar to existing ESC systems; however, unlike conventional ESC, the reference is determined from conditions external to the vehicle, and a simple linear formulation is used. While none of these control modes have been specifically designed to address the path deviation problem, comparisons can be made to understand the operation of the optimal control sequence which is shown as controller (d) in Figs 3 to 5.

Results are shown in Figs 3 to 5, where, as expected, the optimal strategy gives the smallest lateral deviation in each case. The optimal control is seen to be capable of curving the path of the vehicle mass centre back towards the road centre. It is significant that the performance of the three simple strategies is inconsistent between the three cases; for example in case 1, the zero braking control performs badly, while in case 2 it is the most successful of the simple strategies in limiting path lateral deviations.

The results are now considered in more detail. In case 1, Fig. 3 shows a common tendency for the path to curve away from the desired initial trajectory; clearly the yaw rate after impact may generate lateral tyre forces that amplify the disturbance, and this effect is greatest when the wheels are freely rolling, so without braking (a) the deviation is also greatest. By contrast the locked wheel (c) provides an almost straight-line path and a greatly reduced path deviation. The optimal intervention (d) closely mimics the locked wheel result until later in the PI response, then releasing the wheels to provide certain desirable path curvature, and this happens when the sign of the vehicle side slip is reversed (relative to the reversed orientation of the vehicle) allowing the vehicle to steer back towards the initial path. The simple conclusion from Fig. 3 is that choosing a suitable strategy for brake actuation during the immediate PI event can have a very significant effect on lateral deviation from the initial path - reducing the worstcase (free-rolling) deviation by around 75 per cent, from $10.56 \mathrm{~m}$ to $2.83 \mathrm{~m}$. Combined with the discussion of section 1, this supports the conjecture that appropriate brake action applied immediately after an initial impact has the potential to greatly reduce the probability of a serious secondary collision.

Table 1 PI initial conditions of studied cases (side impact from the right, $\nu_{\mathrm{PI}}=15 \mathrm{~m} / \mathrm{s}, \beta_{\mathrm{PI}}=15^{\circ}, \psi_{\mathrm{PI}}=0^{\circ}, \mu=0.9$ )

\begin{tabular}{llrr}
\hline Case & First impact location & $\dot{\psi}_{\mathrm{PI}}\left({ }^{\circ} / \mathrm{s}\right)$ & $\alpha_{f \mathrm{PI}}\left({ }^{\circ}\right)$ \\
\hline 1 & forward CoG & 143 & 22 \\
2 & Behind CoG & -143 & 5 \\
3 & Slightly forward CoG & 57 & -1 \\
4 & at CoG & 0 & 17 \\
\hline
\end{tabular}




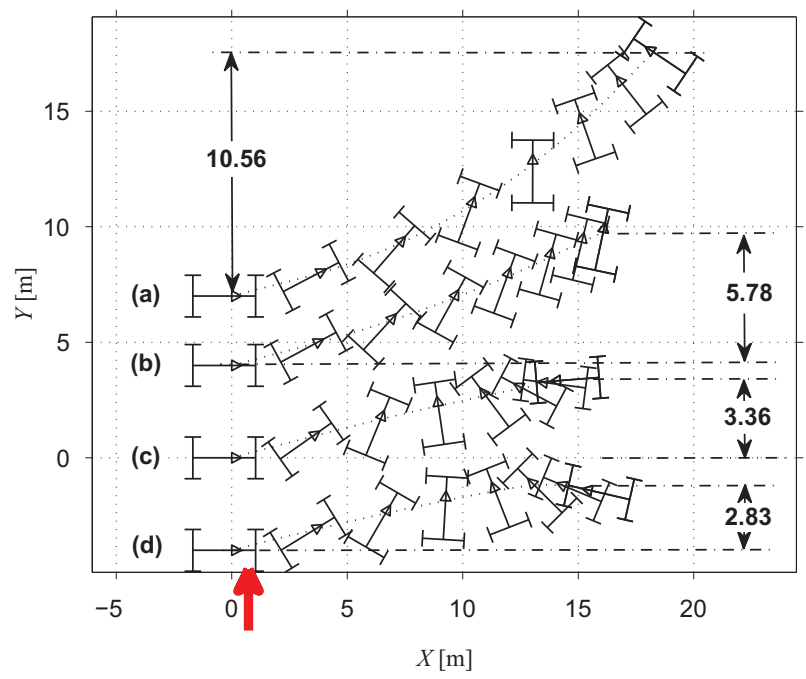

Fig. 3 Case 1: vehicle path of four control strategies: (a) zero braking, (b) yaw control via differential braking, (c) full braking to wheel lock, (d) optimal strategy. Large red arrow approximates the first impact force. (Time interval: $0.26 \mathrm{~s}$.)

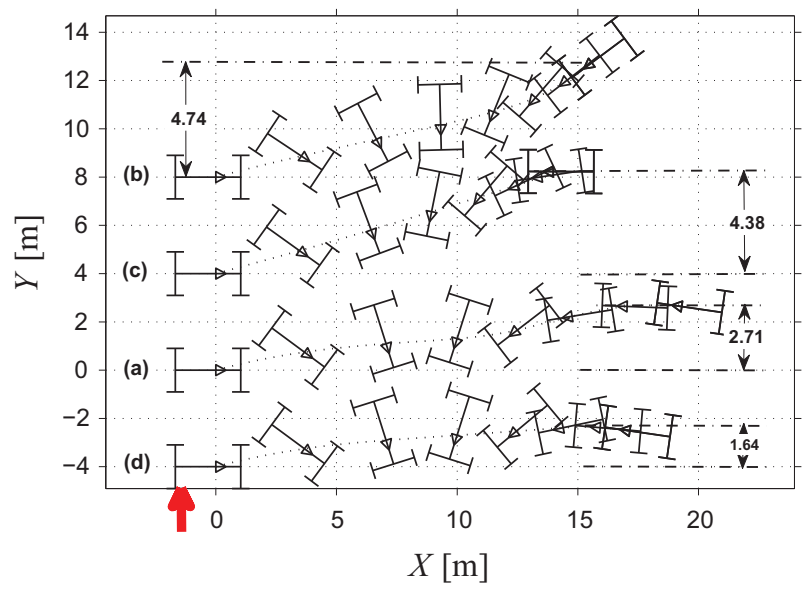

Fig. 4 Case 2: vehicle path of four control strategies: (a) zero braking, (b) yaw control via differential braking, (c) full braking to wheel lock, (d) optimal strategy. Large red arrow approximates the first impact force (time interval: $0.26 \mathrm{~s}$.)

In case 2 (Fig. 4) a very different set of responses is seen; the typical lateral deviation is reduced, with path curvature to the right reducing the effect of the impact. It is seen that the full braking (locked wheel) control negates this desirable path curvature and is one of the worst strategies; the worst path is actually seen for the yaw control strategy, so the response that does most to stabilize the yaw motion actually has the worst effect on path. Comparing (a) and (d)

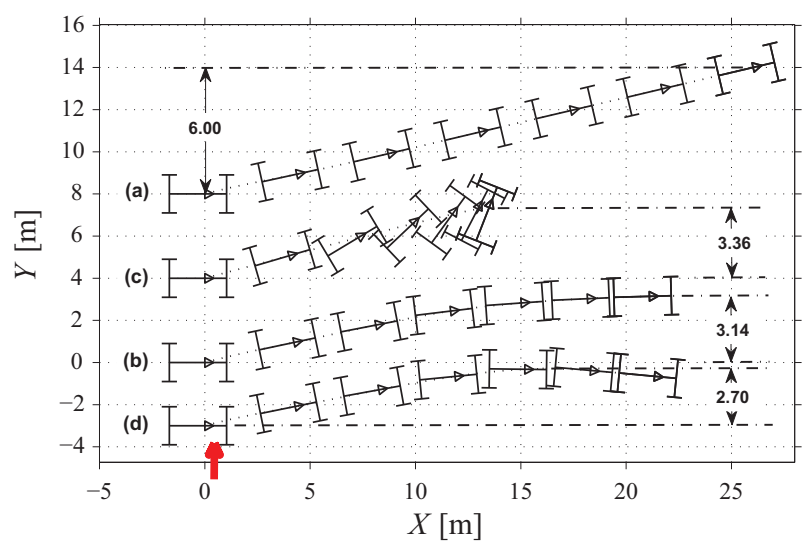

Fig. 5 Case 3: vehicle path of four control strategies: (a) zero braking, (b) yaw control via differential braking, (c) full braking to wheel lock, (d) optimal strategy. Large red arrow approximates the first impact force. (Time interval: $0.30 \mathrm{~s}$.)

in Fig. 4, it is clear that the optimal strategy is very similar to the free-rolling case, at least for the first $10 \mathrm{~m}$ of travel. In this example it is clear that the vehicle kinematics after impact generate lateral tyre forces that oppose the disturbance, and the effect is greatest for free rolling wheels. Again the optimal braking control significantly reduces the maximum lateral deviation, by around 65 per cent compared to the worst case.

In case 3, where the initial yaw rate is small (Fig. 5) the yaw control strategy (b) is the best of the three simple strategies, and comes close to the performance of the optimal intervention, even though it has no direct feedback of lateral offset. By contrast, the free-rolling case (a) performs very badly and full braking limits path deviation mainly by slowing the vehicle down - both (a) and (c) experience straight paths, unlike the more successful interventions.

In the above examples, the optimal use of brake actuators often approximates those of the simple control modes. To understand how these vehiclelevel behaviours emerge, and better characterize the general operation of the optimal controller, attention is now turned to the resultant forces and moments acting on the vehicle.

\section{IDENTIFICATION OF VEHICLE LEVEL CONTROL STRATEGIES}

The resultant force acting at the vehicle mass centre is conventionally resolved in vehicle-oriented coordinates. Here however, with the potential for large yaw rates and yaw angles (as in cases 1 and 2) it is 


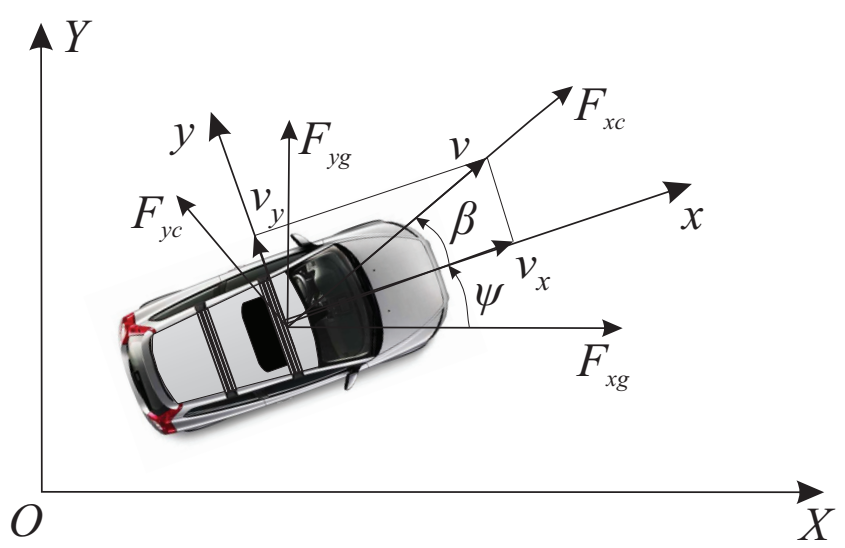

Fig. 6 Definition of coordinates, velocities and forces (illustration of vector directions but not magnitude relations)

more convenient and instructive to use force components based on the particle motion of the mass centre. Two natural options exist: path-based $\left[F_{x c}, F_{y c}\right]^{\mathrm{T}}$ aligned parallel and perpendicular to the instantaneous mass centre velocity vector, or globally based $\left[F_{x \mathrm{~g}}, F_{y \mathrm{~g}}\right]^{\mathrm{T}}$ relative to axes $O X Y$ where $O X$ is the intended path orientation (see Fig. 6).

A possible advantage of using path-based coordinates is that resultant path-lateral force capacity is independent of the vehicle heading angle, and the applied path-lateral force directly determines the local path curvature. On the other hand, use of global axes takes direct account of the optimization goal, and has the simplifying property that $F_{y \mathrm{~g}}(t)$ uniquely determines the value of the cost function (equation (3)); $F_{x \mathrm{~g}}(t)$ can affect the longitudinal distance travelled, but it plays no role in the control performance and is essentially eliminated from the analysis of optimal control. Thus vehicle forces are resolved in the global $O X Y$ axes and focus on $F_{y g}$ as the dominant variable to determine the control of path deviations (zero steer angle being assumed)

$$
F_{y \mathrm{~g}}=\sum_{i}\left(F_{x i} \sin \psi+F_{y i} \cos \psi\right)
$$

As noted, $M_{z}$ influences the overall $F_{y \mathrm{~g}}(t)$ force capacity via the yaw kinematics

$$
\begin{aligned}
M_{z}= & l_{\mathrm{f}} \cdot\left(F_{y \mathrm{fl}}+F_{y \mathrm{fr}}\right)-l_{\mathrm{r}} \cdot\left(F_{y \mathrm{rl}}+F_{y \mathrm{rr}}\right) \\
& +\frac{t}{2} \cdot\left(F_{x \mathrm{fr}}-F_{x \mathrm{fl}}\right)+\frac{t}{2} \cdot\left(F_{x \mathrm{rr}}-F_{x \mathrm{rl}}\right)
\end{aligned}
$$

Therefore the relative contributions of $F_{y g}$ and $M_{z}$ during the above PI cases is of critical interest in describing the operation of the optimal control sequences found in section 3 . In the following subsections three distinct vehicle-level strategies can be identified for which the contributions of $F_{y \mathrm{~g}}$ and $M_{z}$ are optimal.

\subsection{Lateral force control}

Further detail for case 1 is shown in Fig. 7. Plot (a) shows the vehicle motion as well as the available and actual tyre forces during the post impact event; the solid blue line represents the force magnitude and direction at each tyre, and the purple lines map out a sector of attainable tyre forces, assuming the individual braking forces were varied. These available tyre forces are also aggregated to find the set of resultant forces $F_{y \mathrm{~g}}$ and yaw moments $M_{z}$ available at any instant. Of particular interest is the largest available $F_{y g}$ that opposes the current path deviation (i.e. having maximum component in the direction opposing $Y$ )

$$
F_{y \mathrm{~g}}^{*}(t)=\min _{F_{x i}}\left(\operatorname{sgn}(Y(t)) \cdot F_{y \mathrm{~g}}(t)\right)
$$

This is plotted as the dot-dashed curve in Fig. 7 (b) together with the force $F_{y g}$ delivered by the optimal brake control (solid line). In this case, $F_{y \mathrm{~g}}^{*}$ is mostly achieved during the event: the solid and dotdashed lines are close together $(0<X<14 \mathrm{~m})$, preceding the maximum lateral deviation $Y_{\max }$. Some discrepancy is seen soon after the initial impact $(0<X<4 \mathrm{~m})$ where the available limit is not achieved. The corresponding behaviour for case 2 in Fig. 8 is even simpler: for $0<X<14 \mathrm{~m}$ the solid line tracks the dash-dot line very accurately, so the optimal strategy is to instantaneously maximize $F_{y g}$ throughout this extended period of PI response.

Figures 7(b) and 8 show additional information in the dashed and dotted curves, which respectively indicate the contribution to $F_{y g}$ from the longitudinal and lateral forces at the tyres. Thus for instance in case 2, the free-rolling mode persists for $0<X<8.5$ $\mathrm{m}$ (Fig. 8) as all the corrective forces derive from lateral tyre forces. In case 1 the lateral tyre forces mostly act in the 'wrong' direction $(2<X<10 \mathrm{~m})$, i.e. harmful contributions are made to $F_{y \mathrm{~g}}$, acting in the same sense as the original first impact, so locking the wheels reduces the path deviation.

Even though very different braking actions are seen in the above cases, there is a simple dominant strategy operating for most time instants before $Y=Y_{\max }$ : select the individual tyre forces so $F_{y \mathrm{~g}}$ achieves the limiting capacity $F_{y g}^{*}$. From Fig. $7(a)$ it can also be seen how this strategy cascades to the individual brake torques at the wheels; within the sector of available forces, braking torque is applied so the force vector (blue line) has a maximum 


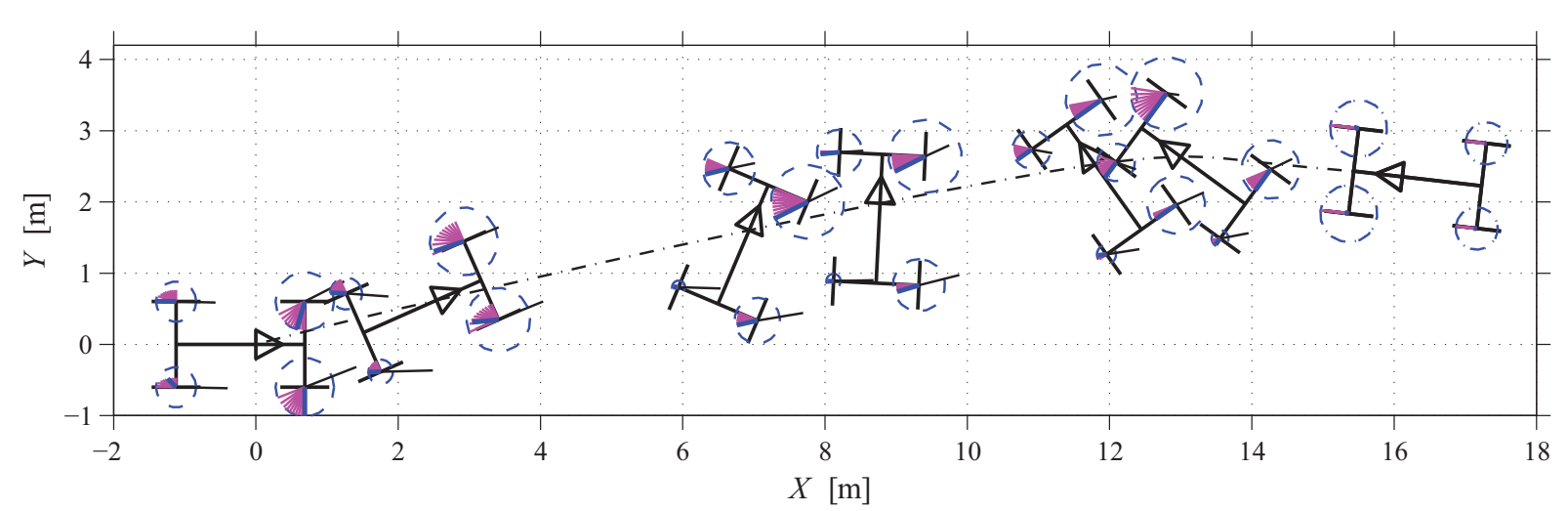

(a)

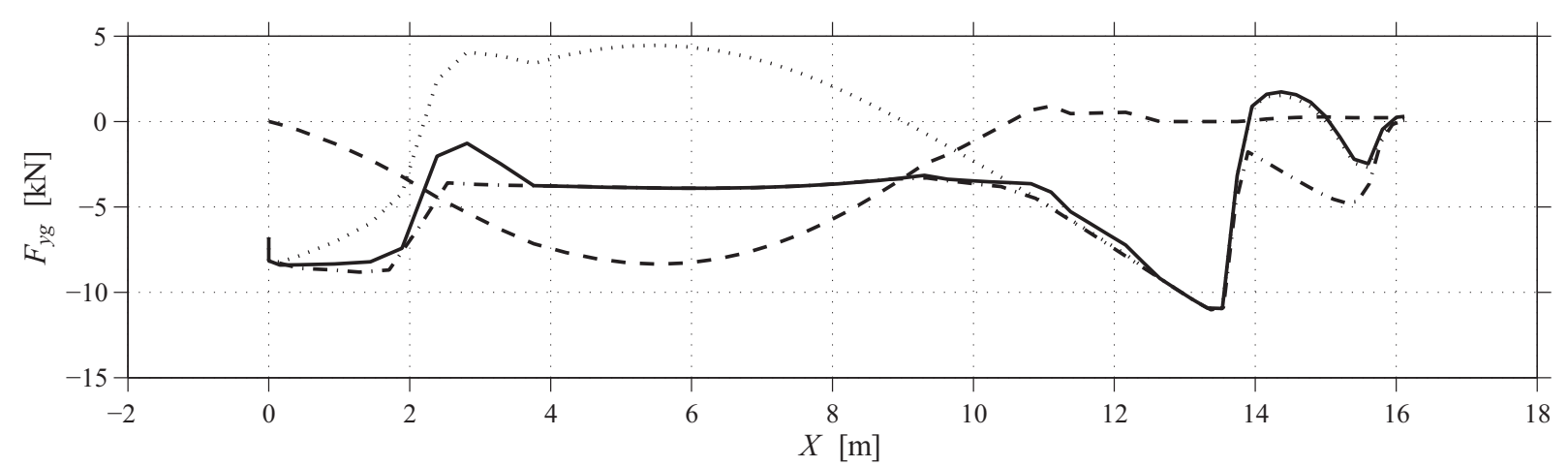

(b)

Fig. 7 Case 1, (a) Optimal path and tyre force vectors (bold: optimal tyre force, thin black: tyre velocity, thin dark grey: available tyre forces, dashed: friction circle). (b) Global lateral forces (solid: resultant lateral force $F_{y g}$, dot-dashed: $F_{y g}^{*}$, dashed: tyre longitudinal force contribution, dotted: tyre lateral force contribution)

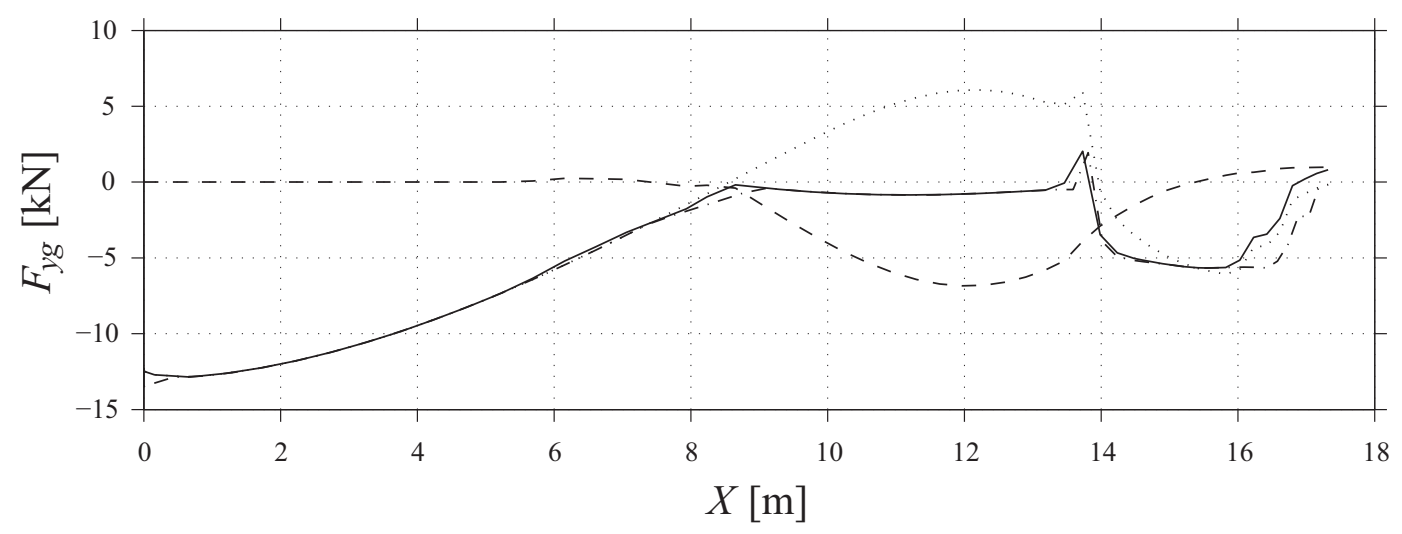

Fig. 8 Case 2, Global lateral forces (solid: $F_{y g}$, dot-dashed: $F_{y g}^{*}$, dashed: tyre longitudinal force contribution, dotted: tyre lateral force contribution)

component in the negative $Y$ direction; this condition holds whenever $F_{y g}=F_{y g}^{*}$ in Fig. 7(a). By contrast, yaw stabilization is far from evident in these two cases. However, this does not imply that $M_{z}$ plays an unimportant role, and it is anticipated that there are times when this variable will provide some critical actions.
To explore utilization and tradeoffs involving $F_{y \mathrm{~g}}$ and $M_{z}$, a 'cloud plot' of available forces and moments is presented in Fig. 9. The available forces and moments are shown as a dark cloud (scatter plot) in the $F_{y \mathrm{~g}}-M_{z}$ plane as the individual brake torque inputs are varied; this method was presented previously in reference [30]. The snapshots of 


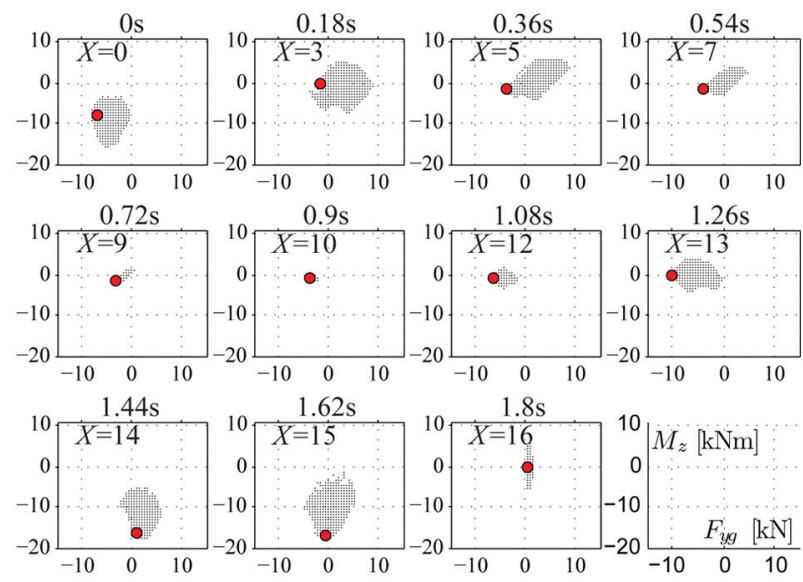

Fig. 9 Case 1, the attainable global lateral force $F_{y g}$ and yaw moment $M_{z}$. The red circle shows the optimal choice

available forces and moments are shown at the discrete braking instances referenced by the optimal control sequence. In each sub-plot the red dot represents the values obtained by optimal braking. As expected, the optimal control corresponds to the point where $F_{y \mathrm{~g}}$ is maximized in the direction of path recovery, i.e. at the leftmost point of the cloud. This is true for most of the critical part of the response, up to and around the maximum path deviation. It is seen that for $X \geqslant 14 \mathrm{~m}$ this is no longer true, but certainly this simple rule dominates the behaviour seen in the clouds. The corresponding plot for case 2 (Fig. 10) shows the same bias to track the leftmost edge of the cloud. It is worth noting here that the choice to use $F_{y \mathrm{~g}}$ rather than $F_{y \mathrm{c}}$ is not critical; the equivalent cloud plots using $F_{y c}$ show very similar cloud shapes and support the same approximate leftmost point rule.
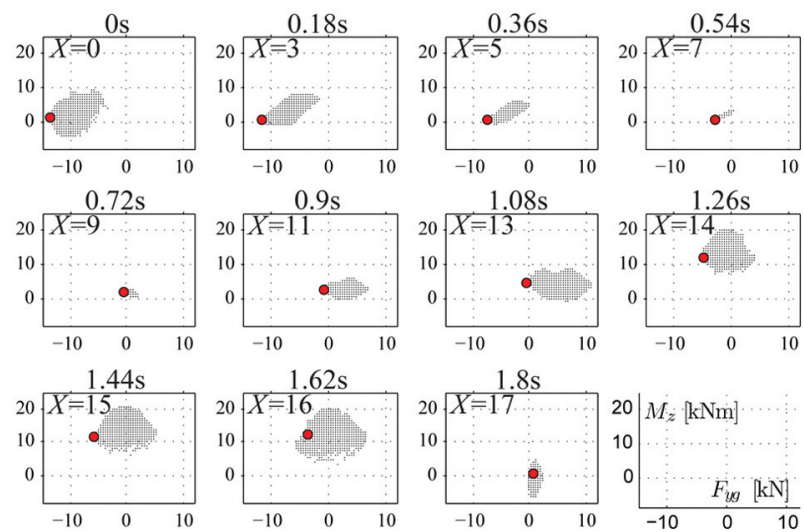

Fig. 10 Case 2, the attainable global lateral force $F_{y g}$ and yaw moment $M_{z}$. The red circle shows the optimal choice
The size and position of the clouds show important features of how the yaw dynamics influences force/moment capacity, mainly via changes in vehicle side slip angle. For instance consider case 2 in Fig. 10: during the interval $0<X<9 \mathrm{~m}$ the cloud migrates from left to the right while at the same time it is shrinking. This interval corresponds to a beneficial vehicle side slip when $F_{y g}$ is easily generated from side forces $F_{y}$ at the tyres. Then, during $9<X<13 \mathrm{~m}$, the clouds expand and move further to the right, indicating the side slip is in the wrong direction so that direct use of braking forces $F_{x}$ is preferred. At the same time the clouds jump to the positive $M_{z}$ axis indicating the availability of yaw moments that force a reduction in rotational energy. Note that when the size of the cloud becomes very small, it corresponds to the instants when $v_{x} \approx 0$ and $\beta \approx 90^{\circ}$ (this is also seen in case 1 - see Figs 9 and 11 (a) for $X \approx 10 \mathrm{~m}$ ), indicating all tyres have slip angles around $90^{\circ}$ and the brake action has little influence on either the path or the yaw dynamics.

Some deviations from the 'leftmost point' rule are evident in the cloud plots of cases 1 and 2, the clearest being during the settling phase in case 1 (see also Fig. 11(a) where for $X \geqslant 14 \mathrm{~m}$ the yaw rate $\dot{\psi}$ rapidly settles towards 0 ). In this case the authors infer that a resisting yaw moment is prioritized, though this is after $Y_{\max }$ is reached and hence with small effect on the cost function and no effect on $Y_{\max }$. In the following, case 3 is considered, which shows a similar exception; however with yaw moment prioritization from the start of the event.

\subsection{Yaw moment control}

Recall that case 3 has a much lower PI initial yaw rate compared to the first two cases, so it is perhaps surprising that yaw moments would play a more important role in this case. Figure 12 shows that for $X<17 \mathrm{~m}$ the global lateral force does not achieve its full capacity, confirming that the optimal strategy is essentially different from cases 1 and 2 . The authors define the yaw moment capacity $M_{z}^{*}$ in a similar way to $F_{y \mathrm{~g}}^{*}$

$$
M_{z}^{*}(t)=\min _{F_{x i}}\left(\operatorname{sgn}(\dot{\psi}(t)) \cdot M_{z}(t)\right)
$$

this being the largest available yaw moment opposing vehicle yaw rotation.

As shown in Fig. 11(b) the yaw rate stabilizes very quickly, with $\dot{\psi}=0$ at around $X=5 \mathrm{~m} ; \dot{\psi}$ then overshoots and returns to zero at around $X=18 \mathrm{~m}$. This generates a positive slip angle $\beta$ during this interval $(0 \leqslant X \leqslant 18 \mathrm{~m})$, leading to a negative lateral acceleration $a_{y}$ and hence a curvature back towards the 

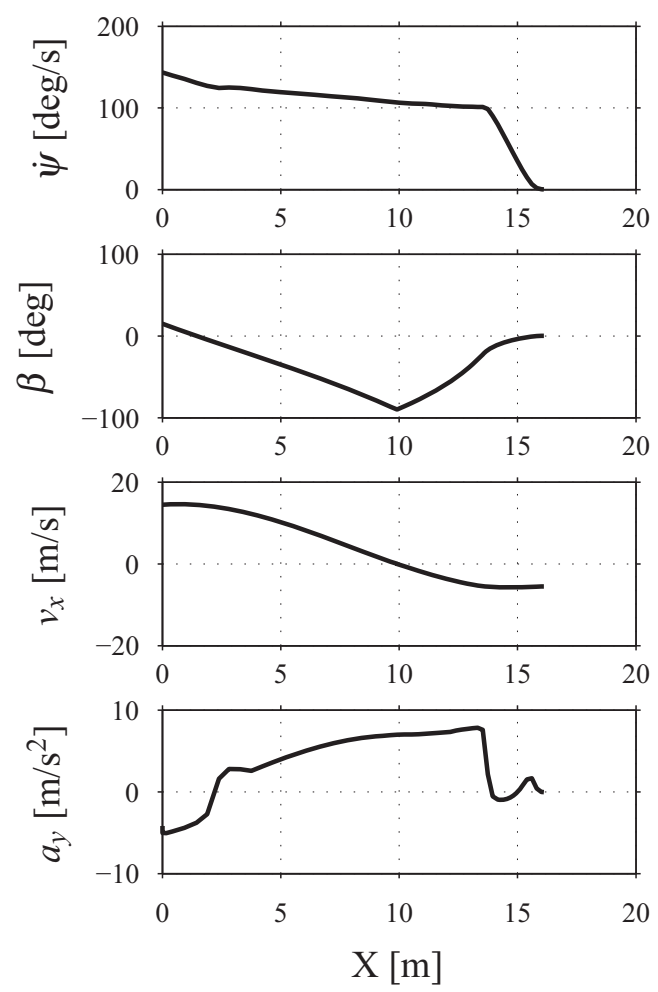

(a) Case 1
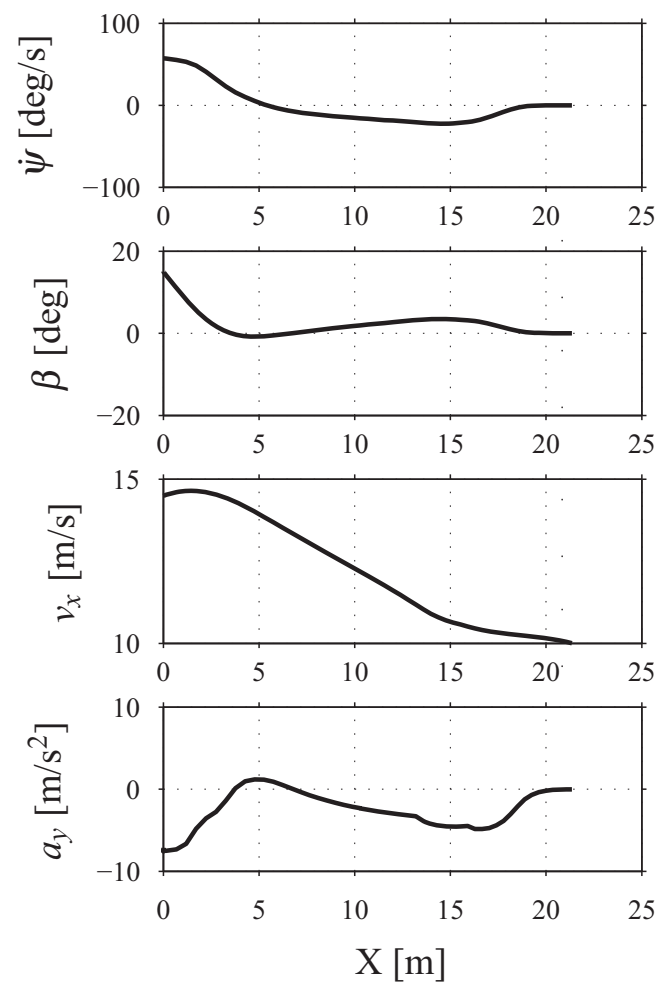

(b) Case 3

Fig. 11 Vehicle motion variables of cases 1 and 3

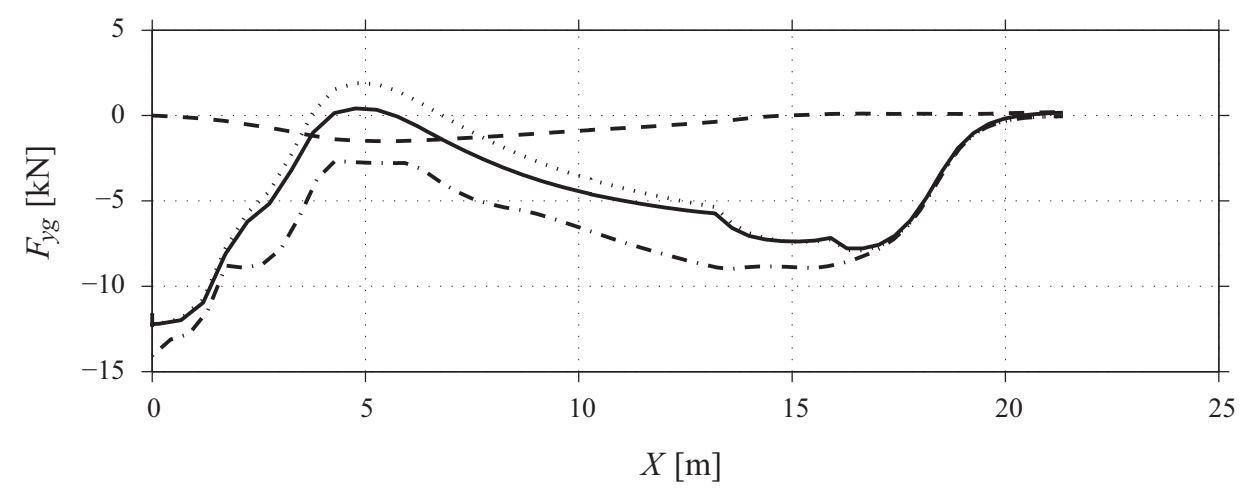

Fig. 12 Case 3, Global lateral forces (solid: $F_{y \mathrm{~g}}$, dot-dashed: $F_{y \mathrm{~g}}^{*}$, dashed: tyre longitudinal force contribution, dotted: tyre lateral force contribution)

desired path. Further, it is noticed that the yaw moment control strategy is able to quickly limit both yaw velocity and side slip angle close to zero values, something that the lateral force control strategy never does.

A similar picture is seen for the global forces in Fig. 13 - a strong negative yaw moment is delivered to the vehicle chassis in preference to achieving $F_{y \mathrm{~g}}^{*}$. Interestingly, the moment remains negative even when the sign of the yaw rate changes, so while $M_{z} \approx M_{z}^{*}$ until $0.54 \mathrm{~s}$, in the subsequent instants, as the chosen point stays near the lower end of the cloud, the yaw moment is actually increasing the magnitude of the yaw rate. Thus this example is seen as a case of yaw moments dominating the character of the optimal response, but not simply providing a yaw stability function.

The cloud plot also shows another basic feature, at least during the early and critical stages of the response when $Y(t)$ is increasing: the selected point is always on or near the left boundary of the cloud, whether or not it is the leftmost point. Equivalently, it may be said that subject to a possible constraint on $M_{z}$, the instantaneous value of $F_{y \mathrm{~g}}$ is at a maximum 


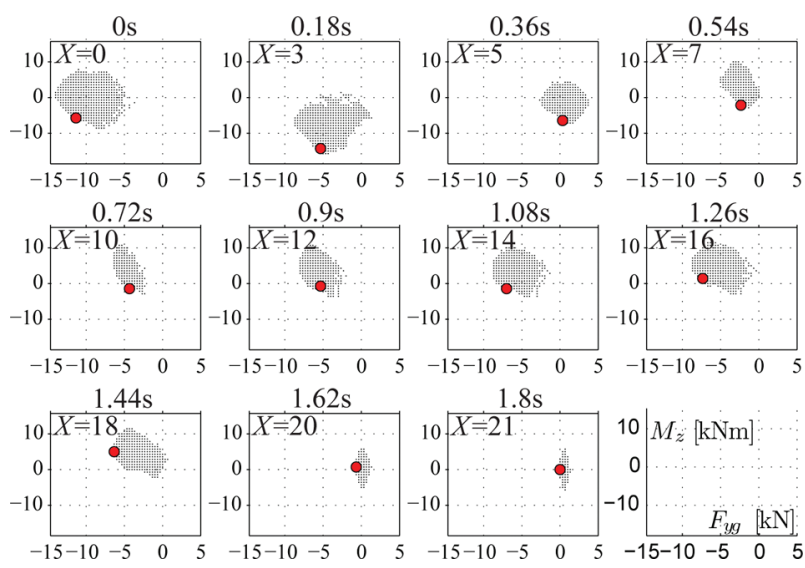

Fig. 13 Case 3, the attainable global lateral force $F_{y g}$ and yaw moment $M_{z}$. The circle shows the optimal choice

opposing $Y$. This simple fact allows the results of all three cases above to be unified $-F_{y g}$ is instantaneously minimized, either with no constraint on $M_{z}$, or with a point constraint determined by the optimizer. This may be formalized as a simple conjecture.

Conjecture: maximum force utilization. In the PI response, for $Y<Y_{\max }$ the optimal response is fully characterized by the yaw moment being either unconstrained, or constrained through values determined by the optimizer. Whether or not the constraint is active, $F_{y \mathrm{~g}}$ achieves its maximum value opposing current $Y$ by appropriate choice of braking torque.

Of course the conjecture is not a proven theorem, and it does not provide an algorithm to compute constraints on $M_{z}$. A broad justification is as follows. Assume $Y(t)$ is optimal but at time $t=t_{1}$ has corresponding points not on the left boundary of the force-moment cloud plot. Assume also that $t_{1}$ precedes the point where $Y=Y_{\max }$. A small improvement in $F_{y \mathrm{~g}}$ can then be achieved for $t_{1}-\varepsilon<t<t_{1}+\varepsilon$ for some small $\varepsilon$, and provided the ensuing forcemoment availability is unaffected the remaining forces and moments can be applied unchanged. If $\tilde{Y}(t)$ denotes the modified path, clearly $\tilde{Y}(t)<Y(t)$ for $t>t_{1}-\varepsilon$ up to $Y=Y_{\max }$, so in this case the modified trajectory is improved. Hence the original trajectory was not optimal because of $F_{y g}$ being interior to the force-moment cloud.

The above argument depends on the cloud plots being insensitive to variations in $F_{y \mathrm{~g}}$, and the conjecture is regarded as a plausible justification for what has been found in the optimization results - any formal proof requires a deeper analysis of the optimization problem, one that will almost certainly require additional assumptions about the vehicle properties and the PI initial conditions. However, if the conjecture is tentatively accepted, it follows that the role of $M_{z}$ is a controlling one. For PI events with large yaw velocities, it has been seen that lateral force control dominates; $M_{z}$ is unconstrained by the path optimization and the appropriate control is achieved by minimizing $F_{y g}$ at each instant. When it becomes feasible to stabilize the yaw rotations, possibly with a reverse orientation of the vehicle, $M_{z}$ dominates the response and the minimization of $F_{y g}$ still applies, but in a constrained sense. Assuming the conjecture is valid, the optimal control problem essentially reduces to defining boundaries and control rules for the yaw moment.

\subsection{Control after point of maximum deviation}

The above argument does not apply once $Y(t)$ is decreasing from its maximum. In that case, the authors expect to see cases where the chosen values of $F_{y \mathrm{~g}}$ and $M_{z}$ are interior to the cloud plot. For completeness case 4 from Table 1 is included, which assumes zero PI yaw rate but non-zero body side slip. The optimal vehicle path indicates a simple type of settling behaviour, qualitatively similar to a damped second-order oscillation in $Y$ (see Fig. 14). In Fig. 15, the selected control (red dot) is clearly inside the cloud boundary when $10<X<13$, which occurs after the point of maximum deviation. Here, as the settling process is near to completion, the size of the cloud is reduced and again the chosen values

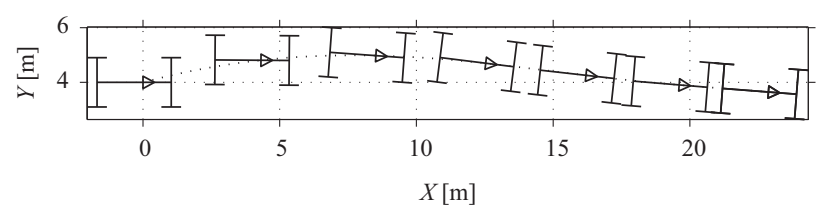

Fig. 14 Case 4, vehicle path
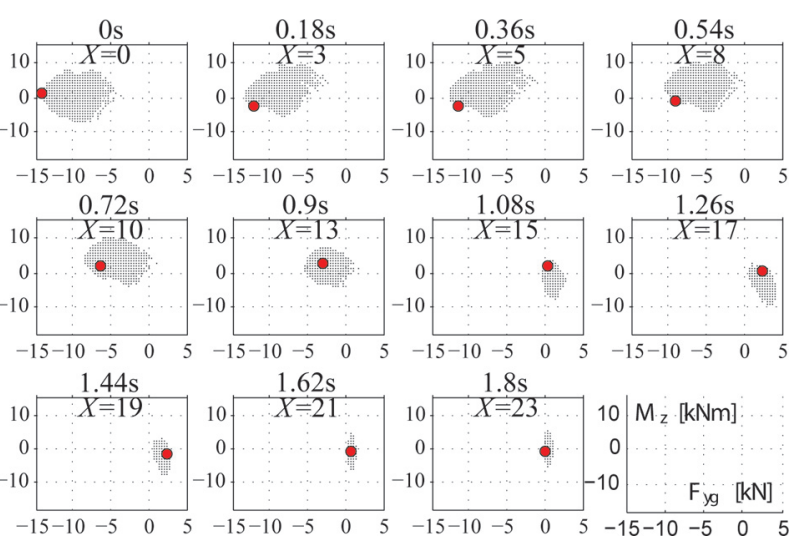

Fig. 15 Case 4 , the attainable global lateral force $F_{y g}$ and yaw moment $M_{z}$. The red circle shows the optimal choice 
are found on the cloud boundaries. Overall the settling has a quasi-linear character similar to a damped second-order system; the dynamics may potentially be analysed using linear optimal control theory; however this aspect of control is clearly of limited importance in the context of the secondary collision avoidance problem, so it is not analysed any further in this paper.

\section{PHASE PLANE ANALYSIS}

According to the above conjecture, force and moment control actions are sometimes constrained by an optimal desired yaw moment, but otherwise dominated by the need to minimize $F_{y \mathrm{~g}}$. This is now tested across a broader range of kinematic conditions by mapping the vehicle-level force/moment control actions onto an appropriate phase plane. A sweep of PI initial yaw rates is considered, maintaining a fixed initial sideslip angle $\beta_{\mathrm{PI}}=15^{\circ}$. For clarity, the phase plane is presented as $\psi$ versus $\dot{\psi}$ (rather than $\psi$ versus $\beta$ ) since yaw velocity defines the initial conditions and yaw angle turns out to be most influential when approaching $180^{\circ}$ or occasionally $360^{\circ}$.

\subsection{Vehicle level control strategies in the phase plane}

The resulting phase portrait is shown by dashed lines in Fig. 16, with $\dot{\psi}_{\text {PI }}$ varying between $-260^{\circ} / \mathrm{s}$ and $+260^{\circ} /$ s. Also shown is colour-coding according to the vehicle-level control strategy adopted at each instant. The dominant colour, red, corresponds to unconstrained minimization of $F_{y g}$, i.e. with the chosen control associated with the left-most point on the instantaneous $F_{y \mathrm{~g}}-M_{z}$ cloud; this is the (lateral) force control strategy seen in section 3 . The other common strategies relate to yaw moment control, which is now resolved into three sub-types, the most common being the stabilizing control (dark blue) when $M_{z} \approx M_{z}^{*}$, i.e. with yaw moment maximizing the dissipation of yaw rotational kinetic energy. There is also seen a destabilizing control (light blue) when $M_{z} \approx-M_{z}^{*}$, i.e. the yaw moment minimizes the dissipation of rotational energy, and the yaw velocity may actually increase in magnitude. The other form of yaw-moment control is called constrained (coloured purple) which instantaneously minimizes $F_{y \mathrm{~g}}$ subject to an implied constraint on $M_{z}$, i.e. with the chosen force/moment combination at the left boundary of the cloud plot, but at a location other than those identified by force, stabilizing or destabilizing control.
The other colours are less directly related to the optimal path control strategy: white indicates the cloud plot is very small (so choice of control action has minimal influence on response), yellow indicates transient force/moment combinations interior to the cloud and green shows points after $Y(t)$ has reached its maximum, i.e. during the settling phase. The noted types of force and moment control actually persist well into the green region, though at the end some linear settling occurs, with points interior to the force-moment cloud, as exemplified by case 4 of section 3 .

Thus, only behaviours seen previously in the four example cases are obtained. The possible exception is the appearance of transient (yellow) regions when force and moment capacity of the vehicle is not fully utilized at each instant during the simulation. Further investigation reveals that this happens when the force/moment capacity (cloud position and shape) changes quickly compared to the discrete sample time $(0.18 \mathrm{~s})$ used for the application of brake control. Indeed, analysis of individual optimizations confirms that these regions occur between discrete sampling instants, and that if the discrete sample time is reduced, the size of these transient regions also reduces; in the limit of a continuoustime controller the yellow regions should be completely eliminated, so within the limited scope and tolerance of the numerical results the above conjecture holds true.

An interesting feature of the phase portrait is that for small (positive) initial yaw rates, the initial response is dominated by stabilizing yaw moment control, and the vehicle settles with a zero yaw angle. Beyond a certain threshold however, the initial response becomes force-controlled (red), switching later on to the stabilizing pattern (blue) and settling into a reversed vehicle orientation $\left(\psi \approx 180^{\circ}\right)$. As the initial yaw rate increases further the rotation goes beyond $180^{\circ}$, tending towards a full $360^{\circ}$ rotation. These qualitative changes in yaw angle during settling occur suddenly as $\dot{\psi}_{\mathrm{PI}}$ smoothly increases, giving a discontinuity in the overall system response; such different responses result in multiple equilibria which are commonly seen in nonlinear dynamics, e.g. reference [31], but here occur because of discrete changes in preference for the optimal controller. The response discontinuity are associated with points of divergence in the phase portrait, including those points indicated by diverging arrows in Fig. 16; two such points are shown, though clearly other cases exist, and further discontinuities occur if the range of initial yaw rates is expanded. Clearly, response discontinuity occurs when it becomes advantageous, at higher rotational 


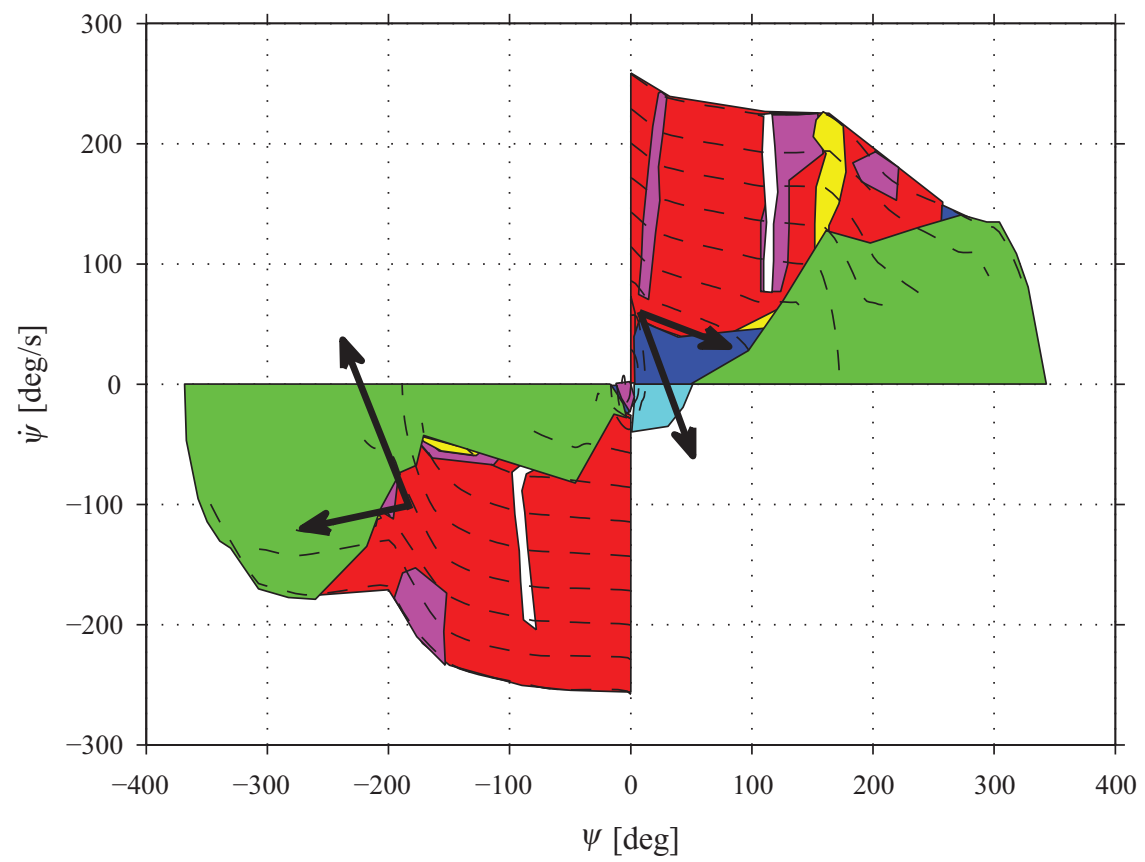

Fig. 16 Optimal vehicle-level control strategies switching in the phase plane. Dashed curves: phase trajectory; red: force control; dark blue: stabilizing; light blue: destabilizing; purple: constrained; white: small cloud condition; yellow: transient; green: settling. Arrows: trajectory divergence directions

energies, to allow greater yaw motion during the initial response while prioritizing instantaneous force control. This is explored further in section 5.3. Note that, even when the response is dominated by force control, the existence of the constrained (purple) regions in the phase plane plot show that small adjustments to the yaw motion are beneficial for limiting the overall path deviation. Attention is now turned to the brake actuation modes associated with the above vehicle-level control strategies.

\subsection{Optimal brake actuation control modes in the phase plane}

In section 3, it was shown that multiple control modes of brake actuation contribute to optimal PI path control; no single brake activation mode, such as locking all wheels, can work well across a wide range of PI conditions. Previously only three braking modes were considered, and the question arises as to whether these suffice under a wider range of conditions. The above optimal responses mapped in the phase-plane, are analysed in terms of which braking mode is active at any particular time. It turns out that a total of five brake actuation modes are required to provide a reasonably complete and non-overlapping map of the optimal control actions. These are shown in Fig. 17, mapped onto the same $\psi-\dot{\psi}$ phase plane of Fig. 16 .
Note that the term Unilateral $D B$ refers to differential braking on either the left or right side of the vehicle, and the term following axle denotes the one currently behind the mass centre, which may be the vehicle's front or rear axle, depending on the sign of $v_{x}$.

The actuator control modes are listed in Fig. 17 and defined via equations (8) to (13), where the thresholds used on the right-hand sides are the result of some tuning, aimed to suitably populate the phase trajectories in a complete and non-overlapping way. The first four modes have been seen previously

$$
\begin{aligned}
& \operatorname{sgn}(\dot{\psi}) \cdot\left(\left(F_{x \mathrm{fl}}+F_{x \mathrm{rl}}\right)-\left(F_{x \mathrm{fr}}+F_{x \mathrm{rr}}\right)\right)>\sum_{i}\left(\mu_{i} F_{z i} \cos \alpha_{i}\right) / 8 \\
& \operatorname{sgn}(\dot{\psi}) \cdot\left(\left(F_{x \mathrm{fr}}+F_{x \mathrm{rr}}\right)-\left(F_{x \mathrm{fl}}+F_{x \mathrm{rl}}\right)\right)>\sum_{i}\left(\mu_{i} F_{z i} \cos \alpha_{i}\right) / 8 \\
& \sum_{i}\left|F_{x i}\right|>\sum_{i}\left(\mu_{i} F_{z i} \cos \alpha_{i}\right) \cdot 0.95 \\
& \sum_{i}\left|F_{x i}\right|<\sum_{i}\left(\mu_{i} F_{z i} \cos \alpha_{i}\right) \cdot 0.05
\end{aligned}
$$

The remaining mode was not seen in the examples of section 3, and it is split into two sub-modes as defined in equation (12) and equation (13). It 


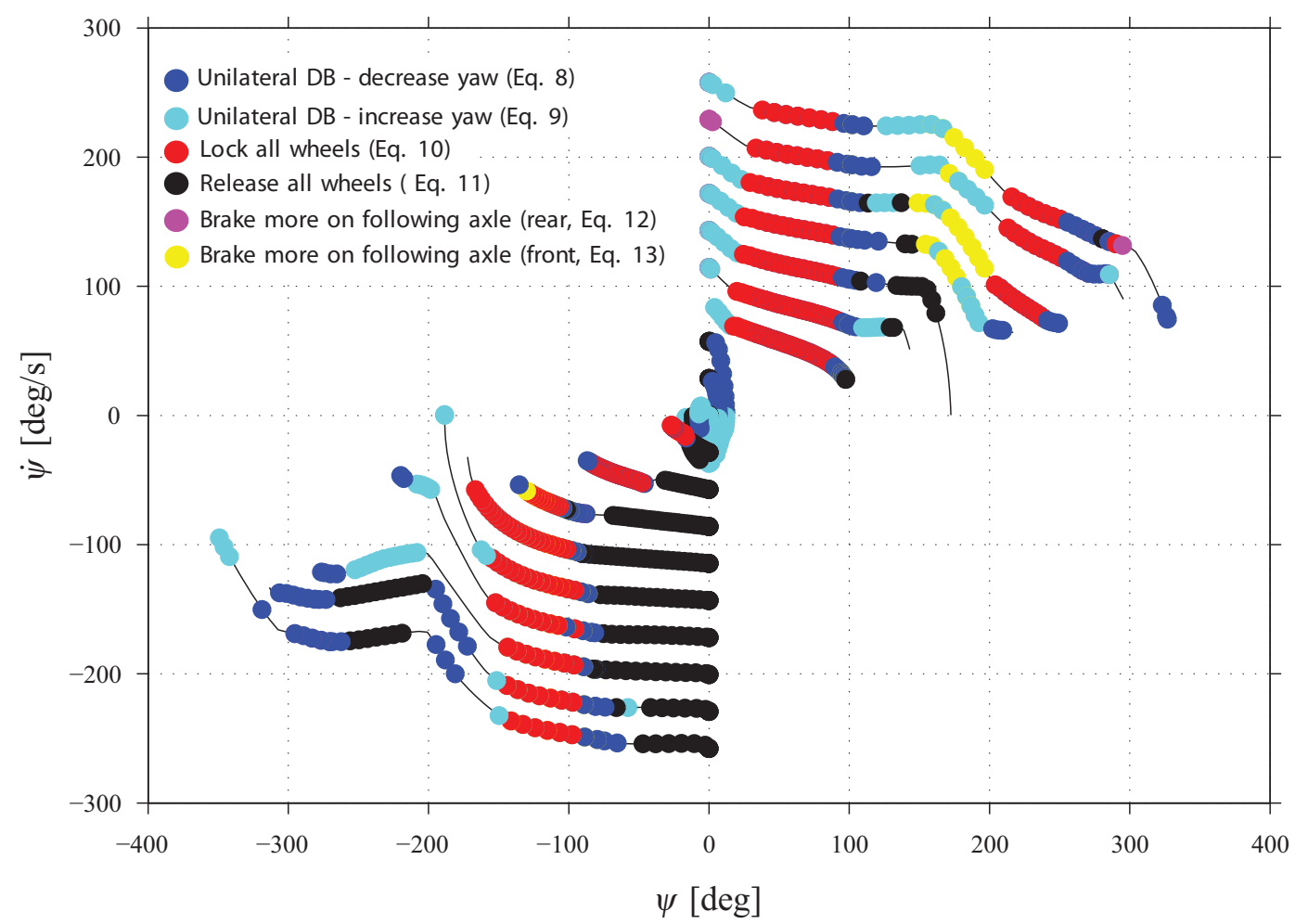

Fig. 17 Brake actuation control modes on phase plane $\left(\beta_{\mathrm{PI}}=15^{\circ}\right)$

applies most braking torque at the following axle (relative to the direction of $v_{x}$ ); this reduces lateral grip on the following axle and hence reduces the stabilizing yaw moment due to lateral tyre forces. For $v_{x} \geqslant 0$, the condition is for increased braking on the rear axle of the vehicle

$$
\left(\left|F_{x \mathrm{rl}}\right|+\left|F_{x \mathrm{rr}}\right|\right)-\left(\left|F_{x \mathrm{fl}}\right|+\left|F_{x \mathrm{fr}}\right|\right)>\sum_{i}\left(\mu_{i} F_{z i} \cos \alpha_{i}\right) / 5
$$

and when $v_{x}<0$, there are more braking at the front axle of the vehicle

$$
\left(\left|F_{x \mathrm{fl}}\right|+\left|F_{x \mathrm{rr}}\right|\right)-\left(\left|F_{x \mathrm{rl}}\right|+\left|F_{x \mathrm{rr}}\right|\right)>\sum_{i}\left(\mu_{i} F_{z i} \cos \alpha_{i}\right) / 5
$$

The results in Fig. 17 include cases similar to those of section 3: for example, in the upper right quadrant, when $\dot{\psi}_{\mathrm{PI}} \in[60,260]^{\circ} / s$ and the same sign as $\beta_{\mathrm{PI}}$, the optimal braking sequence is similar to case 1 ; braking to wheel-lock is mostly applied since $F_{y}$ is harmful until an approximate $90^{\circ}$ yaw angle is reached. When $\dot{\psi}_{\mathrm{PI}} \in[-260,-20]^{\circ} / s$, having the opposite sign to $\beta_{\mathrm{PI}}$, it is similar to case 2 , with zero-braking initially dominating. When $\dot{\psi}_{\mathrm{PI}} \in[-20,60]^{\circ} / s$, the yaw disturbance is sufficiently mild and the response is similar to case 3 , with stabilizing yaw moment control prioritized until $\psi \approx 0^{\circ}$. Overall, it is seen that the optimal response involves switching among the five simple brake actuation modes. Importantly, these modes can be obtained directly from the simpler vehiclelevel strategies mentioned above, using information about the ins-tantaneous vehicle kinematics; for example, in the unconstrained case when braking torques are chosen to minimize $F_{y g}$, the direction of each wheel-force vector is to align as closely as possible with $-Y$, and hence each braking torque can be computed.

\subsection{Discontinuous response and multiple equilibria}

As noted in section 5.1, when the initial yaw rate is gradually increased in magnitude, a discontinuity in response takes place, with a discrete switching of the dominant control strategy from stabilizing yaw moment control to lateral force control. One such discontinuity is shown in greater detail in Fig. 18; this corresponds to the pair of arrows in the upper right quadrant in Fig. 16. Four phase trajectories are shown, two below the point of discontinuity and two above. Note that, although the response is discontinuous as $\dot{\psi}_{\text {PI }}$ crosses its threshold, the value of the optimal cost remains continuous. Further analysis of this particular event shows that there is no specific dynamic transition, such as tyre force saturation, associated with the response discontinuity. Rather, the discontinuity occurs at a crossover 


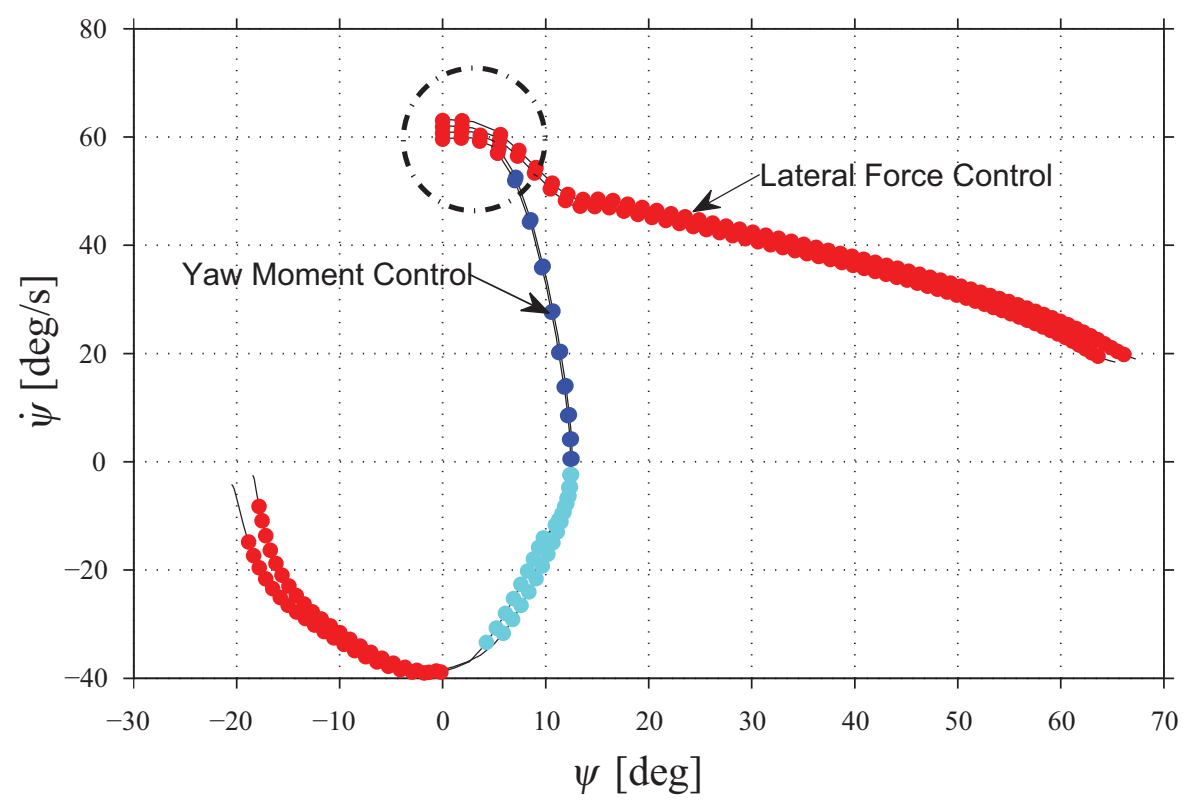

Fig. 18 Discontinuous response on phase plane, $\dot{\psi}_{\mathrm{PI}}>0, \psi_{\mathrm{PI}}=0^{\circ}, \beta_{\mathrm{PI}}=15^{\circ}, \nu_{\mathrm{PI}}=15 \mathrm{~m} / \mathrm{s}$ (same colour codes as in Fig. 16)

point, when the lateral force control becomes advantageous, and this is consistent with the continuity of the cost function.

Furthermore, this discontinuity can be characterized by the equilibrium, i.e. yaw angle to which the vehicle settles, where multiple equilibria are seen. In Fig. 18 the responses below the threshold settle towards $\psi=0^{\circ}$, while those above the threshold move towards another equilibrium: $\psi=180^{\circ}$. This is clearer in Fig. 17 where as the initial yaw rate increases the phase trajectories progressively tend towards $\psi=180^{\circ}$, until eventually there is enough initial rotational energy to trigger a second discontinuity and settling towards $\psi=360^{\circ}$. With greater initial yaw velocity it would clearly be possible to trigger third and more response discontinuities, though such cases are expected to be only of theoretical interest.

A more general mapping of the response discontinuity at post impact initial conditions is shown in Fig. 19, where the PI side slip $\beta_{\mathrm{PI}}$ is also allowed to vary. The case of Fig. 18 is represented by the circle. It is worth noting that for $\beta_{\mathrm{PI}}=0^{\circ}$, the upper and lower points of discontinuity are symmetrical, as should be expected for a laterally symmetric vehicle. As $\beta_{\text {PI }}$ increases, between $10^{\circ}$ to $60^{\circ}$, the points of discontinuity become insensitive to the value of $\beta_{\mathrm{PI}}$, and the choice of dominant strategy is mainly dependent on initial yaw velocity. With even higher PI side slip, the region of yaw moment control shrinks. As seen in section 4.2, the yaw moment control strategy is used when it can stabilize both the yaw rate and side slip simultaneously. In the case of large $\beta_{\mathrm{PI}}$, clearly this stabilization is harder to achieve, hence the lateral force control strategy is selected at very low values of $\dot{\psi}_{\mathrm{PI}}$.

\section{CONCLUSIONS}

This study has shown that appropriate brake actions can be made to control the per impact trajectory of a vehicle involved in a light collision, so that in principle the probability of a secondary impact or road departure can be reduced. In the examples presented, the particular choice of braking actions has a significant effect on the vehicle path. An optimal control analysis has been developed, based on a fourth power cost function of the path deviation lateral to the vehicle intended path; the function provides a satisfactory approximation to the problem of minimizing the maximum path deviation.

No single simpler control mode (e.g. yaw motion control or locked-wheel braking) was found to be optimal in reducing lateral deviations. Instead, within one accident, dynamic switching between a combination of these modes appears to be necessary. Only in some post impact circumstances, a locking of all four wheels gives lateral deviation close to be optimal.

The optimal response is found to include a wide variety of brake actuator control modes, ranging from locking all wheels to allowing all wheels to roll freely. The mode chosen at any instant is dependent on the vehicle-level force and moment capability, especially the force capability orthogonal to the intended path. In turn, the global lateral force capability depends heavily on vehicle orientation and slip angle - i.e. the instantaneous force capability is 


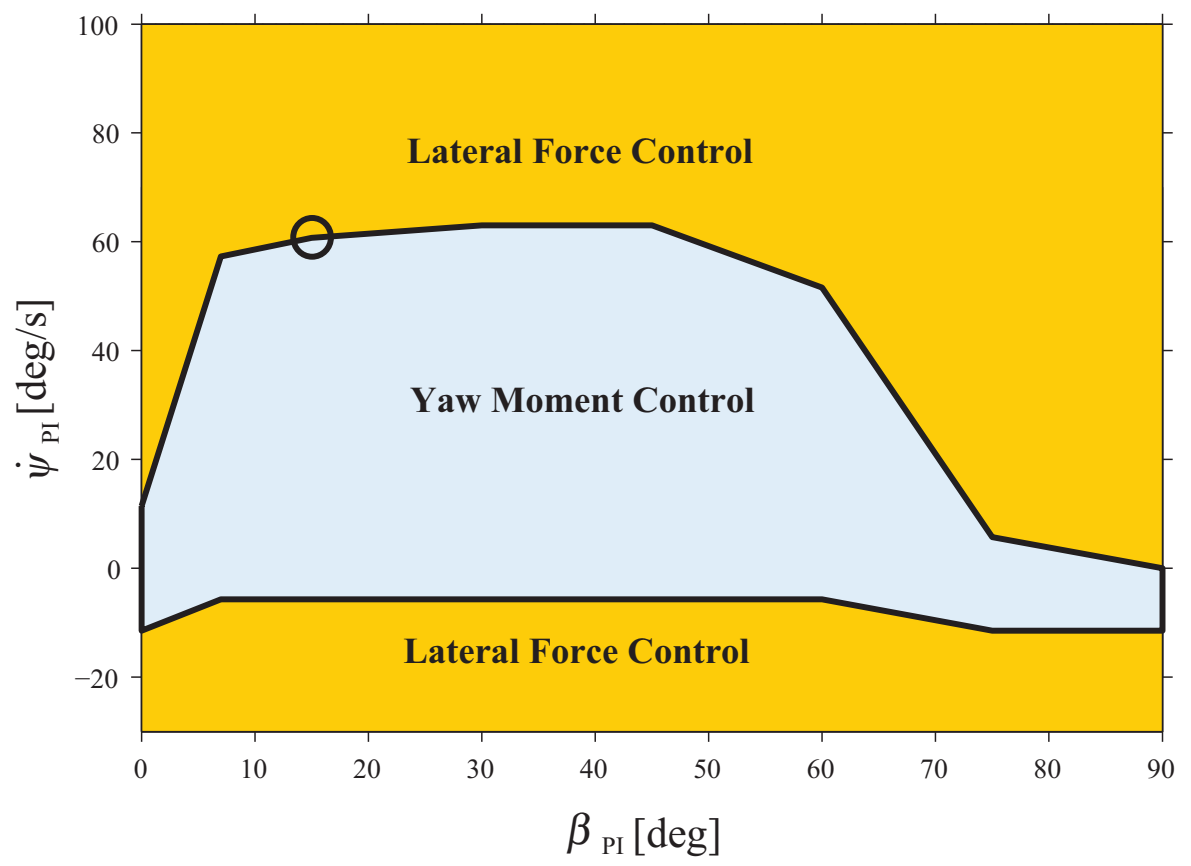

Fig. 19 Discontinuous response at PI initial conditions, $\psi_{\mathrm{PI}}=0^{\circ}, v_{\mathrm{PI}}=15 \mathrm{~m} / \mathrm{s}$ (circle corresponds to the one in Fig. 18)

highly anisotropic, if no other actuators than the friction brakes are at present.

Three vehicle level control strategies have been identified for optimal path control: (i) global lateral force is maximized at every instant in the direction opposing harmful lateral deviations; (ii) yaw moment control is applied, operating at its upper or lower limit, or choosing an intermediate value that in turn constrains the available lateral forces - in this case the most favourable global lateral force is applied; and (iii) a settling motion takes place that involves synchronized contributions from lateral force and yaw motion, and forces and moments are not on the limits. In terms of the clouds plots of available forces and moments, these strategies were identified as being on the cloud boundary - (i) and (ii) - or in the interior region (iii). These control strategies were seen to be optimal across a wide range of PI conditions, the explanation being given in the form of a general conjecture, and validation given via a phase plane analysis.

The phase plane analysis also demonstrated the common occurrence of discontinuities between responses respectively dominated by global lateral force control and yaw moment control. Overall, five actuator modes were identified as being prevalent. Future work is required to establish the feasibility of a robust closed-loop strategy that incorporate these actuator modes and vehicle-level strategies for implementation on the vehicle.
In this paper, the lateral displacement is measured at the mass centre, and additional lateral deviations due to yaw motion are not taken into account. Of course the paths of corner points of the vehicle body are especially important for assessing collision risk, and it could be argued that it is the envelope of these paths that should be used. The envelope [32] is a curve tangent to each path, and bounds their combined maximum path deviations. This is relevant because the impending secondary collision can happen to any corner of the vehicle. On the other hand this increases the complexity of the optimization and the interpretation of results, so the authors avoided it in this analysis, simplifying the requirement to that of mass centre path deviation. It is noted here that attitude (i.e. yaw angle) control is also influential for crash risk, and also affects the likely severity of a crash outcome. Future studies for reducing crash risk and severity in secondary collisions should include objective functions for both lateral position and yaw angle. Specific case studies will also be needed to evaluate system performance for real-world post impact scenarios.

\section{FUNDING}

This work was supported by the Vehicle and Traffic Safety Centre (SAFER) at Chalmers University of Technology [grant number AD3], and Volvo Car Corporation, Göteborg [grant number TSU-nr27807].

(C) Authors 2011 


\section{REFERENCES}

1 Sander, U., Mroz, K., Boström, O., and Fredriksson, R. The effect of pre-pretensioning in multiple impact crashes, 2009, Autoliv Research, Sweden, paper no. 09-0333, pp. 1-11.

2 Yang, D., Jacobson, B., and Lidberg, M. Benefit prediction of passenger car post impact stability control based on accident statistics and vehicle dynamics simulations. In Proceedings of the 21st International Symposium on Dynamics of vehicles on roads and tracks, Stockholm, Sweden, 2009.

3 Fay, P., Sferco, R., and Richard, F. Multiple impact crashes - consequences for occupant protection measures. In Proceedings of the IRCOBI Conference, Isle of Man, UK, 2001.

4 Langwieder, K., Sporner, A., and Hell, W. RESICO - retrospective safety analysis of car collisions resulting in serious injuries, GDV9810, Munich, Germany, 1999.

5 Zhou, J., Lu, J., and Peng, H. Vehicle stabilisation in response to exogenous impulsive disturbances to the vehicle body. Int. J. Vehicle Autonom. Syst., 2010, 8(2/3/4), 242-262.

6 Sander, U. Requirements to occupant restraint systems in multiple impact crashes in-depth analysis of real world accident data. Master's Thesis, June 2009, Technical University in Graz, Graz, Austria (to be published 2012).

7 Yang, D. Method for benefit prediction of passengercar post impact stability control. Master's Thesis, Chalmers University of Technology, Gothenburg, Sweden, 2009.

8 Van Zanten, A. ESP electronic stability program in Robert Bosch GmbH, 2nd edition, 1999, pp. 206-243 (Society of Automotive Engineers, Warrendale, PA).

9 Koibuchi, K., Yamaoto, M., and Fukada, Y. Vehicle dynamics control in limit cornering by active brake. SAE technical paper 960487, 1996.

10 Naraghi, M., Roshanbin, A., and Tavasoli, A. Vehicle stability enhancement - an adaptive optimal approach to the distribution of tyre forces. Proc. IMechE, Part D: J. Automobile Engineering, 2010, 224(4), 443-453.

11 Yi, K., Chung, T., Kim, J., and Yi, S. An investigation into differential braking strategies for vehicle stability control. Proc. IMechE, Part D: J. Automobile Engineering, 2003, 217(12), 1081-1093.

12 Van Zanten, A. T. Evolution of electronic control systems for improving the vehicle dynamic behavior. In Proceedings of the 6th International Symposium on Advanced vehicle control, Hiroshima, Japan, 2002.

13 Salfeld, M., Stabrey, S., and Trächtler, A. Optimal control inputs to affect vehicle dynamics in various driving states. In Proceedings of the 7th IFAC Symposium on Nonlinear control systems, Pretoria, South Africa, 2007.

14 Robert Bosch GmbH Secondary collision mitigation: protection against subsequent crashes. 21 September 2010, available from http://rb-kwin.bosch. $\mathrm{com} / \mathrm{hu} / \mathrm{en} /$ safety_comfort/drivingsafety/capsco mbinedactivepassivesafety/occupantprotection/sec ondarycollisionmitigation.html.
15 Tan, H.-S. and Chan, C.-Y. Design of steering controller and analysis of vehicle lateral dynamics under impulsive disturbances. In Proceedings of the American Control Conference, Chicago, IL, June 2000, pp. 2023-2027.

16 Volvo Car Corporation: safety preventive. 1 June 2010, available from http://www.volvocars.com/ us/sales-services/sales/Documents/Volvo-2010-XC 60-brochure.pdf.

17 Kritayakirana, K. and Gerdes, J. C. Autonomous cornering at the limits: designing a longitudinal feedback controller using a tire slip circle. In Proceedings of the 10th International Symposium on Advanced vehicle control, Loughborough, UK, 2010, pp. 418-423.

18 Rajamani, R. Vehicle dynamics and control, 2006 (Springer, Berlin).

19 Seiler, P., Song, B., and Hedrick, J. K. Development of a collision avoidance system. SAE paper 980853, 1998.

20 Jula, H., Kosmatopoulos, E. B., and Ioannou, P. A. Collision avoidance analysis for lane changing and merging. IEEE Trans. Vehicul. Technol., 2000, 49(6), 2295-2308.

21 Blank, M. and Margolis, D. L. Minimizing the path radius of curvature for collision avoidance minimizing the path radius of curvature for collision avoidance. Vehicle Syst. Dynam., 2000, 33(3), 183-201.

22 Gehrig, S. K. and Stein, F. J. Collision avoidance for vehicle-following systems. IEEE Trans. Intell. Transport. Syst., 2007, 8(2), 233-244.

23 Su, J.-L. and Ordys, A. W. Collision avoidance manoeuvre for a vehicle - a practical approach. Proc. IMechE, Part D: J. Automobile Engineering, 2009, 224(3), 299-312.

24 Gordon, T. J. and Best, M. C. Lyapunov control of vehicle handling dynamics. JSAE Rev., 1999, 20, 453-458.

25 Furukawa, Y. and Abe, M. Advanced chassis control systems for vehicle handling and active safety. Vehicle Syst. Dynam., 1997, 28(2), 59-86.

26 Pacejka, H. B. Tire and vehicle dynamics, 2nd edition, 2006 (Society of Automotive Engineers, Warrendale, PA).

27 Yang, D., Gordon, T., Lidberg, M., Jonasson, M., and Jacobson, B. Post-impact vehicle path control by optimization of individual wheel braking sequences. In Proceedings of the 10th International Symposium on Advanced vehicle control, Loughborough, UK, 2010, pp. 882-887.

28 The MathWorks, documentation, optimization toolbox, functions. 11 October 2009, available from http:/ / www.mathworks.com/access/helpdesk/help/ toolbox/optim/ug/fmincon.html.

29 Bauer, H. Bosch automotive handbook, 6th edition, 2004 (Society of Automotive Engineers, Warrendale, PA).

30 Jonasson, M., Andreasson, J., Jacobson, B., and Trigell, A. S. Global force potential of over-actuated vehicles. Vehicle Syst. Dynam., 2010, 48(9), 983-998.

31 Khalil, H. K. Nonlinear systems, 3rd edition, 2002 (Prentice-Hall, Englewood Cliffs, NJ).

32 Envelop (mathematics). 11 October 2010, available from http://en.wikipedia.org/wiki/Envelope. 


\section{APPENDIX 1}

\section{Notation}

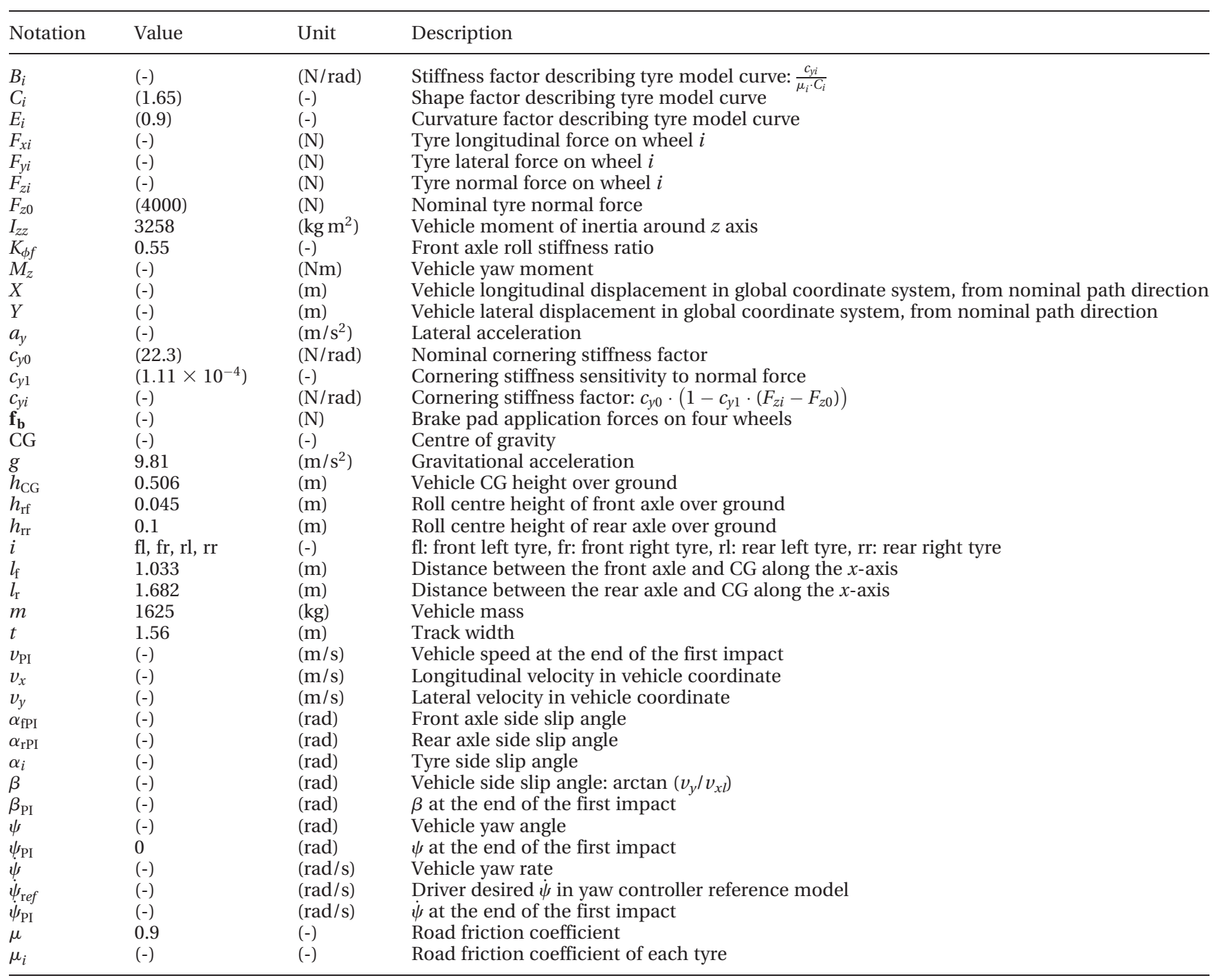

\section{APPENDIX 2: YAW MOTION CONTROLLER}

The yaw motion controller is a proportional-integral one with constant gains. The braking force requirement is linearly proportional to yaw rate error and yaw angle error. The proportional coefficient is $K_{p}$, and the integral coefficient is $K_{i}$ which are tuned for the exemplar cases in the paper. No optimal controller with wheel slip control so as to maximize yaw correction moment is intended to be designed for this paper. In this study the reference yaw rate is zero, since straight road and fixed zero steering angle are assumed. Both tyres on one side of the car are braked, and the sign of required yaw moment $M_{z}$ decides the side and thus the braking force distribution on four wheels $\mathbf{f}_{\mathbf{b}}$. The friction braking force on the tyre-road contact patch $F_{x i}$ is opposite to the tyre longitudinal velocity. The control law is shown in equations (14) and (15)

$$
\begin{aligned}
& M_{z}=\operatorname{sgn}\left(v_{x}\right) \cdot\left(-\mathrm{K}_{\mathrm{p}}\left(\dot{\psi}-\dot{\psi}_{\mathrm{ref}}\right)-\mathrm{K}_{\mathrm{i}}\left(\int_{0}^{t}\left(\dot{\psi}-\dot{\psi}_{\mathrm{ref}}\right) \mathrm{d} t\right)\right)
\end{aligned}
$$

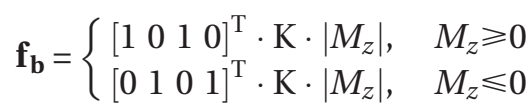

where $K_{\mathrm{p}}=1 \cdot 10^{5} \quad[\mathrm{Nm} /(\mathrm{rad} / \mathrm{s})], \quad K_{\mathrm{i}}=2 \cdot 10^{5} \quad[\mathrm{Nm} /$ $\mathrm{rad}], \mathrm{K}=1[1 / \mathrm{m}]$. 